\title{
Radiation and MHD Boundary Layer Stagnation-Point of Nanofluid Flow towards a Stretching Sheet Embedded in a Porous Medium: Analysis of Suction/Injection and Heat Generation/Absorption with Effect of the Slip Model
}

\author{
Emad H. Aly ${ }^{1,2}$ \\ ${ }^{1}$ Department of Mathematics, Faculty of Science, King Abdulaziz University, Jeddah 21589, Saudi Arabia \\ ${ }^{2}$ Department of Mathematics, Faculty of Education, Ain Shams University, Roxy, Cairo 11757, Egypt
}

Correspondence should be addressed to Emad H. Aly; efarag@kau.edu.sa

Received 9 March 2015; Accepted 8 May 2015

Academic Editor: Gen Qi Xu

Copyright (C) 2015 Emad H. Aly. This is an open access article distributed under the Creative Commons Attribution License, which permits unrestricted use, distribution, and reproduction in any medium, provided the original work is properly cited.

In existence of the velocity slip model, suction/injection, and heat source/sink, the boundary layer flow near a stagnation-point over a heated stretching sheet in a porous medium saturated by a nanofluid, with effect of the thermal radiation and magnetic field, has been studied. The governing system of partial differential equations was transformed into a system of nonlinear ordinary equations using the appropriate similarity transforms. Then, the obtained system has been numerically solved by the Chebyshev pseudospectral differentiation matrix (ChPDM) approach. It was found that, at some special cases, the current results are in a very good agreement with those presented in the literature. In addition, the flow velocity, surface shear stress, temperature, and concentration are strongly influenced on applying the slip model, which is, therefore, extremely important to predict the flow characteristics accurately in the nanofluid mechanics. It was proved that this velocity slip condition is mandatory and should be taken into account in nanoscale research; otherwise, false results and a spurious physical sight are to be gained. Further, it was deduced that the influence of the stream velocity and shear stress reaches very rapidly the stable manner for both cases of the velocity ratio. However, when this ratio is equal to one, the skin friction coefficient, reduced Nusselt number, and reduced Sherwood number are constant and equal to zero, 0.721082, and 3.06155, respectively. Furthermore, it was proved that the reduced Nusselt number decreases with increase of Brownian motion and thermophoresis; has a very weak effect on increasing Lewis number; increases with increase of Prandtl number; and is higher in the cases of suction, velocity ratio $>1$ and heat source in comparison with injection, velocity ratio $<1$, and heat sink, respectively. Moreover, the reduced Sherwood number increases with increase of Brownian motion, thermophoresis, and Lewis number; decreases with increase of Prandtl number; is higher in the cases of suction and velocity ratio $>1$ in comparison with injection and velocity ratio $<1$, respectively; and is approximately the same in the heat source and heat sink cases. Finally, it was shown that the most effective region for radiation effect is $[0,1]$.

\section{Introduction}

Applications on the Stretching Sheet Study. The problem of flow and heat transfer in boundary layer over a stretching surface has attracted many researchers because of its numerous applications, for example, in metallurgical processes, such as drawing of continuous filaments through quiescent fluids, annealing and tinning of copper wires, glass blowing, manufacturing of plastic and rubber sheets, crystal growing, and continuous cooling and fiber spinning. In addition, there are wide-ranging applications in many engineering processes, such as polymer extrusion, wire drawing, continuous casting, manufacturing of foods and paper, glass fiber production, and stretching of plastic films. During the manufacture of these sheets, the melt issues from a slit and is subsequently stretched to achieve the desired thickness. The final product 
with the desired characteristics strictly depends upon the stretching rate, the rate of cooling in the process, and the process of stretching.

Nanofluids and Their Applications. Nanotechnology is nowadays considered as a significant factor which affects the industrial revolution of the current century. Nanofluids are nanometer-sized particles (diameter less than $50 \mathrm{~nm}$ ) dispersed in a base fluid. Recent research on nanofluids showed that nanoparticles changed the fluid characteristics because thermal conductivity of these particles was higher than convectional fluids. It should be noticed that nanoparticles are of great scientific interest as they are effectively a bridge between bulk materials and atomic or molecular structures. These nanoparticles are typically made of metals, oxides, carbides, or carbon nanotubes. The common base fluids include water, ethylene glycol, toluene, and oil. Therefore, many researchers have focused on modeling the thermal conductivity and examined different types of nanofluids' viscosity (see $[1,2])$.

Convective heat transfer in nanofluids is a topic of major contemporary interest both in applied sciences and engineering. Choi [3] may be the first author to use the term "nanofluid" in his seminal paper presented in 1995 at the ASME Winter Annual Meeting, where it was reported that one of the promising nanofluids applications is heat transfer enhancement. In [4], Choi et al. showed that the addition of a small amount (less than $1 \%$ by volume) of nanoparticles to conventional heat transfer liquids increased the thermal conductivity of the fluid up to approximately two times. Masuda et al. [5], Lee et al. [6], Xuan and Li [7], and Xuan and Roetzel [8] stated that, with low nanoparticles concentrations (1-5 Vol\%), the thermal conductivity of the suspensions can increase more than $20 \%$. Regarding, some interest has been given to the study of this type of flow and some useful results have been recently introduced by many authors; see, for example, [9-13]. Aly and Ebaid [14] presented a comprehensive study of convective transport in nanofluids. Buongiorno [15] developed an analytical model for convective transport in nanofluids considering the Brownian diffusion and thermophoresis. He developed an explanation for abnormal convective heat transfer enhancement observed in nanofluids and also showed that Brownian diffusion and thermophoresis were the most important nanoparticle/basefluid slip mechanisms.

Many of the publications on nanofluids are about the understanding of their behaviors so that they can be utilized where straight heat transfer enhancement is paramount as in many industrial applications, nuclear reactors, transportation, electronics, and biomedicine and food. Examples include nanofluid adhesive: electronics cooling, vehicle cooling, transformer cooling, super powerful and small computers cooling, and electronic devices cooling; medical applications: cancer therapy and safer surgery by cooling and process industries; and materials and chemicals: detergency, food and drink, oil and gas, paper and printing, and textiles. Ultra high-performance cooling is necessary for many industrial technologies. However, poor thermal conductivity is a drawback in developing energy-efficient heat transfer fluids necessary for ultra high-performance cooling.

Investigations on the Stagnation-Point. In 1911, Hiemenz was the pioneer to analyze two-dimensional stagnation-point flow on stationary plate using a similarity transformation to reduce the Navier-Stokes equations to nonlinear ordinary differential equations. Due to its wide range of applications in cooling of electronic devices by fans, cooling of nuclear reactors during emergency shutdown, solar central receivers exposed to wind currents, and many hydrodynamic processes in engineering application, the flow near the stagnation-point has attracted the attention of many investigators for more than a century.

The idea of stagnation-point flow of a nanofluid is extended and studied recently. Accordingly, Mahapatra and Gupta [16] and Ishak et al. [17] studied numerically boundary layer and magnetohydrodynamics stagnation-point flow towards a stretching sheet. Their analysis showed that velocity at a point increases with an increase in the magnetic field when the free stream velocity is greater than the stretching velocity. Layek et al. [18] have reported heat and mass transfer boundary layer stagnation-point flow of an incompressible viscous fluid towards a heated porous stretching sheet embedded in a porous medium subject to suction/blowing with internal heat generation or absorption. The governing boundary layer equations were transformed by scaling group of transformation into a system of ordinary differential equations. Moreover, Bachok et al. [19] expanded the dimension of the problem of stagnation-point flow and heat transfer to three-dimensional stagnation-point flow in nanofluids. Mustafa et al. [20] studied the stagnation-point flow of a nanofluid towards a stretching sheet using homotopy analysis method. To follow up some deducing properties in [18], Hamad and Ferdows [21] studied heat and mass transfer for boundary layer stagnation-point flow over a stretching sheet in a porous medium saturated by a nanofluid with internal heat generation/absorption and suction/injection. They deduced that the inclusion of nanoparticles into the base fluid of this problem changes the flow pattern, where suction tends to stabilize the boundary layer flow and blowing can reduce the friction drag [18].

Recently, a good physical explanation of nanofluid stagnation-point flow and radiation heat transfer over a stretching sheet with first-order velocity slip and temperature jump in a porous medium has been introduced by Zheng et al. [22]. Further, Ibrahim et al. [23] analyzed magnetohydrodynamics stagnation-point flow towards a stretching sheet in nanofluid. They found that the magnitude of the skin friction coefficient, the reduced Nusselt number, and reduced Sherwood number are all increasing with the magnetic parameter when the velocity ratio exceeds 1 , and when this ratio is less than 1 they all decrease. In addition, the heat transfer rate at the surface increases with velocity ratio and Prandtl number. Likewise, the mass transfer rate at the surface increases with an increase in both Lewis number and velocity ratio. Very recently, Jalilpour et al. [24] studied the MHD boundary layer flow of a nanofluid towards a stretching surface with suction or blowing and surface heat 
flux. They found that the magnitude of the reduced Nusselt number decreases with an increase in magnetic number, thermophoresis parameter, and Lewis number. Further, the reduced Sherwood number decreases with increasing magnetic number and thermophoresis parameter and increases with increasing Lewis number. Furthermore, the reduced Nusselt number and reduced Sherwood number are higher when suction is induced.

Importance of the Slip Model in Nano Studies. In 1997, Thompson and Troian [25] proved that the slip velocity is related to the slip length, the shear rate at the wall, and a critical shear rate at which the slip length diverges. In addition, wall slip readily occurs for an array of complex fluids such as foams, emulsions, suspensions, and polymer solutions. Further, the fluids that exhibit boundary slip have important technological applications such as in the polishing of artificial heart valves and internal cavities. Later, Majumder et al. [26] showed experimentally that nanofluidic flow usually exhibits partial slip against the solid surface, which can be characterized by the so-called slip length (around 3.4-68 micrometers for different liquids). They proved that the classical no-slip condition is no longer valid for fluid flows at the microand nanoscale and, instead, a certain degree of tangential slip must be allowed. Hence, effect of partial slip boundary condition on the flow and heat transfer of nanofluids past stretching sheet at constant wall temperature has been studied by Noghrehabadi et al. [27] to extend the work done by Khan and Pop [28]. In addition, Nandeppanavar et al. [29] have tabulated the literature of the first-order slip, and this resulted in making Fang et al. [30] considered only the effect of the second-order slip on the flow on a shrinking sheet. Therefore, they may be the first researchers to investigate the analysis of the second-order slip flow and heat transfer over a stretching sheet using Wu's slip model [31]. Further, Turkyilmazoglu [32] has analytically studied the heat and mass transfer of magnetohydrodynamic second-order slip flow. He has mentioned that there exists a unique solution for any combination of the considered parameters if the stretching sheet is considered. Furthermore, Roşca and Pop [33] investigated the steady flow and heat transfer over a vertical permeable stretching/shrinking sheet with secondorder slip. This very important study showed clearly that the second-order slip flow model is necessary to predict the flow characteristics accurately.

To prove the point of view in [33], Aly and Ebaid [14] have recently investigated five nanofluids flow over an isothermal stretching sheet with effect of Wu's slip model [31]. They showed that increase of the slips slows down the velocity, increases the temperature with an impressive effect in the injection case, and decreases the local skin friction and the reduced Nusselt number with significant effects. Recently, Aly and Vajravelu [34] examined the effects of the second-order slip parameter for the nano boundary layer flow over two-dimensional and axisymmetric stretching surfaces in the presence of a transverse magnetic field in a porous medium. They found that this parameter affects considerably the flow characteristics for increasing values of the magnetic parameter and decreasing values of the porosity parameter. In addition, the presence of the magnetic field, permeability, first and second slip factors lead to a decrease in the nano boundary layer thickness. Very recently, Aly and Hassan [35] investigated the dynamic effects including the Brownian motion and thermophoresis, the developments of the second-order slip velocity on the boundary layer flow and heat transfer over a stretching surface in the presence of nanoparticle fractions. They proved that the second-order slip parameter influences strongly the flow velocity and surface shear stress on the stretching sheet and also the reduced Nusselt and the reduced Sherwood numbers.

Aim of the Present Work. The purpose of the present work is therefore to analyze the boundary layer stagnation-point flow towards a stretching sheet in a porous medium saturated by a nanofluid in suction/injection cases and in the presence of radiation, magnetic field, and heat generation/absorption with effect of the velocity slip boundary condition. The mathematical formulation of the current model is introduced in Section 2, where proper similarity transforms are then applied to gain a system of nonlinear ordinary differential equations which represents the flow through the boundary layer. The resulting system is numerically solved by the Chebyshev pseudospectral differentiation matrix (ChPDM) in Section 3 and is then discussed via tables and figures in Section 4 , besides comparison with already published results.

\section{The Mathematical Modelling}

2.1. Description of the Problem. In this research, we consider a steady laminar two-dimensional flow of a viscous incompressible electrically conducting nanofluid near a stagnationpoint over a heated stretching sheet in a porous medium, which is saturated by a nanofluid coinciding with the plane $y=0$, where the flow region is confined to $y>0$. It is assumed that two equal and opposite forces are applied along the $x$-axis regarding the surface is stretched where the position of the origin is fixed [18]. In addition, the nanofluid is investigated under the effect of thermal radiation and a uniform magnetic field of strength $B_{0}$ is applied in the positive direction of $y$-axis. However, the magnetic Reynolds number is assumed to be small, that the induced magnetic field is negligible [24]. Further, the velocities of the external flow and stretching sheet are $u_{e}(x)=a x$ and $u_{w}(x)=b x$, respectively, where $a$ and $b$ are positive constants.

2.2. Basic Equations. In the presence of heat source/sink and with neglecting the viscous dissipation, the basic conservation of mass, momentum, thermal energy, and nanoparticles equations for nanofluids, which match the above physical model, can be written in the Cartesian coordinates $x$ and $y$ as

$$
\begin{aligned}
\frac{\partial u}{\partial x}+\frac{\partial v}{\partial y} & =0 \\
u \frac{\partial u}{\partial x}+v \frac{\partial u}{\partial y} & =u_{e} \frac{\partial u_{e}}{\partial x}+\nu \frac{\partial^{2} u}{\partial y^{2}}+\left(\frac{v}{k}+\frac{\sigma B_{0}^{2}}{\rho}\right)\left(u_{e}-u\right),
\end{aligned}
$$




$$
\begin{aligned}
u \frac{\partial T}{\partial x}+v \frac{\partial T}{\partial y}= & \alpha \frac{\partial^{2} T}{\partial y^{2}}+\frac{Q_{0}}{\rho c_{p}}\left(T-T_{\infty}\right) \\
& +\tau\left[D_{B} \frac{\partial \varphi}{\partial y} \frac{\partial T}{\partial y}+\frac{D_{T}}{T_{\infty}}\left(\frac{\partial T}{\partial y}\right)^{2}\right] \\
& -\frac{1}{\rho c_{p}} \frac{\partial q_{r}}{\partial y} \\
u \frac{\partial \varphi}{\partial x}+v \frac{\partial \varphi}{\partial y}= & D_{B} \frac{\partial^{2} \varphi}{\partial y^{2}}+\frac{D_{T}}{T_{\infty}} \frac{\partial^{2} T}{\partial y^{2}}
\end{aligned}
$$

subject to the following boundary conditions:

$$
\begin{aligned}
& \text { at } y=0: \quad u=u_{w}+u_{s}, \quad v=v_{w}, T=T_{w}, \varphi=\varphi_{w}, \\
& \text { as } y \rightarrow \infty: \quad u=u_{e}, T=T_{\infty}, \varphi=\varphi_{\infty},
\end{aligned}
$$

where $u$ and $v$ are the velocity components along the axes $x$ and $y$, respectively, $T$ is the temperature, and $\varphi$ is the volume fraction of nanoparticles. In addition, $\rho$ is the density; $\nu(=\mu / \rho)$ is the kinematic viscosity, where $\mu$ is the dynamic viscosity; $c_{p}$ is the specific heat; $\alpha\left(=\kappa / \rho c_{p}\right)$ is the thermal diffusivity, where $\kappa$ is the thermal conductivity; and $D_{B}$ and $D_{T}$ are the Brownian diffusion and thermophoresis diffusion coefficients, respectively. Further, $k$ is the permeability of the porous medium, $Q_{0}$ is the heat generation or absorption coefficient, $q_{r}$ is the radiative heat flux, $\sigma$ is the electrical conductivity, $\tau$ is the ratio between the effective heat capacity of the nanoparticle material and heat capacity of the fluid (see Khan and Pop [28], Nield and Kuznetsov [36]), and $u_{s}$ expresses the slip velocity model. It should be noted that ()$_{w}$ and ()$_{\infty}$ denote the wall and ambient values, respectively, of the parameters, while ()$_{p}$ refers to the fixed parameter for particles.

Regarding the approximation of Rosseland for radiation (see $[37,38]$ ), the radiative heat flux $q_{r}$ is simplified as

$$
q_{r}=-\frac{4 \sigma^{*}}{3 k^{*}} \frac{\partial T^{4}}{\partial y}
$$

where $\sigma^{*}$ and $k^{*}$ are the Stefen-Boltzmann constant and mean absorption coefficient, respectively. It is assumed that the temperature changes within the flow such that the term $T^{4}$ may be expressed as a linear function of the temperature. Hence, on expanding $T^{4}$ in a Taylor series about $T_{\infty}$ and neglecting the higher order terms, one obtains

$$
T^{4} \cong 4 T_{\infty}^{3} T-3 T_{\infty}^{4}
$$

Therefore, (3) reduces to

$$
\begin{aligned}
u \frac{\partial T}{\partial x}+v \frac{\partial T}{\partial y}= & \alpha \frac{\partial^{2} T}{\partial y^{2}}+\frac{Q_{0}}{\rho c_{p}}\left(T-T_{\infty}\right) \\
& +\tau\left[D_{B} \frac{\partial \varphi}{\partial y} \frac{\partial T}{\partial y}+\frac{D_{T}}{T_{\infty}}\left(\frac{\partial T}{\partial y}\right)^{2}\right] \\
& +\frac{16 \sigma^{*} T_{\infty}^{3}}{3 \rho c_{p} k^{*}} \frac{\partial^{2} T}{\partial y^{2}}
\end{aligned}
$$

2.3. Velocity Slip Model. As mentioned in Section 1, for the continuum modeling of fluidic transport, the assumption of no-slip boundary condition is no longer assumed, and a certain degree of tangential slip must be allowed. In the present work, we consider Wu's slip model which is given by (see [14, 29-31])

$$
\begin{aligned}
u_{s}= & \frac{2}{3}\left(\frac{3-\alpha_{m} l^{3}}{\alpha_{m}}-\frac{3}{2} \frac{1-l^{2}}{K_{n}}\right) \lambda_{m} \frac{\partial u}{\partial y} \\
& -\frac{1}{4}\left[l^{4}+\frac{2}{K_{n}^{2}}\left(1-l^{2}\right)\right] \lambda_{m}^{2} \frac{\partial^{2} u}{\partial y^{2}}=A \frac{\partial u}{\partial y}+B \frac{\partial^{2} u}{\partial y^{2}},
\end{aligned}
$$

where $K_{n}$ is an arbitrary Knudsen number, $l=\min \left[1 / K_{n}, 1\right]$, $0 \leq \alpha_{m} \leq 1$ is the momentum accommodation, and $\lambda_{m}$ is the molecular mean free path. Based on the definition of $l$, it is noticed that, for any given value of $K_{n}$, we have $0 \leq l \leq 1$. Thus the molecular mean free path is always positive. Therefore, the value of $B$ should be negative and hence the second term in the right-hand side of (9) is a positive number.

2.4. Similarity Transforms. The appropriate similarity transforms of (1), (2), (8), and (3) with the boundary conditions (5) are introduced as follows [23]:

$$
\begin{array}{r}
\psi=x \sqrt{b v} f(\eta), \\
\theta(\eta)=\frac{T-T_{w}}{T_{w}-T_{\infty}}, \\
\phi(\eta)=\frac{\varphi-\varphi_{w}}{\varphi_{w}-\varphi_{\infty}}, \\
\text { where } \eta=\sqrt{\frac{b}{v} y .}
\end{array}
$$

The stream function $\psi$ is defined in the usual way as

$$
\begin{aligned}
& u=\frac{\partial \psi}{\partial y}=b x f^{\prime}(\eta) \\
& v=-\frac{\partial \psi}{\partial x}=-\sqrt{b v} f(\eta)
\end{aligned}
$$

to identically satisfy (1). On substituting (10) into (2), (4), and (8), the following ordinary differential equations are obtained:

$$
\begin{aligned}
& f^{\prime \prime \prime}(\eta)+f(\eta) f^{\prime \prime}(\eta)-\left[f^{\prime}(\eta)\right]^{2} \\
& -(M+K)\left[f^{\prime}(\eta)-\lambda\right]+\lambda^{2}=0, \\
& \left(\frac{1}{\operatorname{Pr}}+R\right) \theta^{\prime \prime}(\eta)+f(\eta) \theta^{\prime}(\eta)+\gamma \theta(\eta) \\
& +\operatorname{Nb} \phi^{\prime}(\eta) \theta^{\prime}(\eta)+\operatorname{Nt}\left[\theta^{\prime}(\eta)\right]^{2}=0, \\
& \phi^{\prime \prime}(\eta)+\operatorname{Le} f(\eta) \phi^{\prime}(\eta)+\frac{\mathrm{Nt}}{\mathrm{Nb}} \theta^{\prime \prime}(\eta)=0,
\end{aligned}
$$


subject to the following boundary conditions:

$$
\begin{aligned}
f(0) & =S, \\
f^{\prime}(0) & =1+\delta_{1} f^{\prime \prime}(0)+\delta_{2} f^{\prime \prime \prime}(0), \\
\theta(0) & =1, \\
\phi(0) & =1, \\
f^{\prime}(\infty) & =\lambda, \\
\theta(\infty) & =0, \\
\phi(\infty) & =0
\end{aligned}
$$

where the governing nondimensional parameters are defined as follows: $M\left(=(\sigma / b \nu) B_{0}^{2}\right)$ is the magnetic field, $K(=v / b k)$ is the permeability, $\lambda(=a / b)$ is the velocity ratio, $R(=$ $\left.16 \sigma^{*} T_{\infty}^{3} / 3 \rho c_{p} k^{*}\right)$ is the thermal radiation, $\gamma\left(=Q_{0} / \rho b c_{p}\right)$ is the heat source (when $\gamma>0$ )/sink (for $\gamma<0$ ), $\mathrm{Nb}\left(=\tau D_{B}\left(\varphi_{w}-\varphi_{\infty}\right) / \nu\right)$ is the Brownian motion, and $\mathrm{Nt}\left(=\tau D_{T}\left(T_{w}-T_{\infty}\right) / \nu T_{\infty}\right)$ is the thermophoresis. Further, $\operatorname{Pr}(=\nu / \alpha)$ and Le $\left(=\nu / D_{B}\right)$ are Prandtl and Lewis numbers, respectively, $S\left(=v_{w} / \sqrt{b v}\right)$ is the suction when $S>0$ and injection for $S<0,0<\delta_{1}(=A \sqrt{b / \nu})$, and $0>\delta_{2}(=B b / \nu)$ are the first-order velocity slip and second-order velocity slip, respectively.

It should be noted that, when $\mathrm{Nb}=\mathrm{Nt}=0$, the current study reduces to the classical problem of flow and heat transfer due to a stretching surface in a viscous fluid. In this case, the boundary value problem for $\phi$ becomes illposed without physical significance. Further, as special cases, when $\delta_{1}=\delta_{2}=0$ and with $K=R=\gamma=0$, system (12) to (16) is reduced to that one obtained by Ibrahim et al. [23], with $M=R=\gamma=S=0$, to the system introduced by Hamad and Ferdows [21], and with $R=\gamma=0$ to that given by Jalilpour et al. [24] (on dropping the prescribed surface heat flux). Furthermore, when $M=\gamma=S=\delta_{2}=0$, the present model matches that one obtained by Zheng et al. [22] (with absence of the temperature jump).

2.5. Quantities of Practical Interest. In this type of study, it should be noted that the quantities of practical interest are the skin friction coefficient $C_{f}$, local Nusselt number $\mathrm{Nu}_{x}$, and local Sherwood number $\mathrm{Sh}_{x}$, which are defined as

$$
\begin{aligned}
C_{f} & =\frac{\tau_{w}}{\rho u_{w}^{2}}, \\
\mathrm{Nu}_{x} & =\frac{x q_{w}}{\kappa\left(T_{w}-T_{\infty}\right)}, \\
\mathrm{Sh}_{x} & =\frac{x q_{m}}{D_{B}\left(\phi_{w}-\phi_{\infty}\right)},
\end{aligned}
$$

where $\tau_{w}, q_{w}$, and $q_{m}$ are the skin friction (or shear stress), heat flux from the surface, and mass flux, respectively, which are given by

$$
\begin{aligned}
& \tau_{w}=-\mu\left(\frac{\partial u}{\partial y}\right)_{y=0}, \\
& q_{w}=-\kappa\left(\frac{\partial T}{\partial y}\right)_{y=0}, \\
& q_{m}=-D_{B}\left(\frac{\partial \phi}{\partial y}\right)_{y=0} .
\end{aligned}
$$

Therefore, on using (11) in (17) and (18), we obtain

$$
\begin{aligned}
C_{f}\left(\operatorname{Re}_{x}\right)^{1 / 2} & =f^{\prime \prime}(0), \\
\operatorname{Nur} & =\operatorname{Nu}_{x}\left(\operatorname{Re}_{x}\right)^{-1 / 2}=-\theta^{\prime}(0), \\
\operatorname{Shr} & =\operatorname{Sh}_{x}\left(\operatorname{Re}_{x}\right)^{-1 / 2}=-\phi^{\prime}(0),
\end{aligned}
$$

where $\operatorname{Re}_{x}\left(=b x^{2} / v\right)$ is the local Reynolds number based on the stretching velocity, Nur and Shr are referred as reduced Nusselt number and reduced Sherwood number as mentioned by Khan and Pop [28] and then by many others; see, for example, Vajravelu et al. [39], Hamad and Ferdows [21], and Aly and Ebaid [14].

\section{Numerical Approach}

In order to solve the coupled ordinary differential equations (12)-(14), which are third order in $f$ and second order in both $\theta$ and $\phi$, with the boundary conditions (15) and (16), we apply the Chebyshev pseudospectral differentiation matrix (ChPDM) technique. Hence, these equations become

$$
\begin{aligned}
& \sum_{j=0}^{N} d_{i, j}^{(3)} f\left(z_{j}\right)+f\left(z_{i}\right)\left(\frac{\eta_{\infty}}{2}\right) \sum_{j=0}^{N} d_{i, j}^{(2)} f\left(z_{j}\right) \\
& -\left(\frac{\eta_{\infty}}{2}\right)\left(\sum_{j=0}^{N} d_{i, j}^{(1)} f\left(z_{j}\right)\right)^{2} \\
& -(M+K)\left(\frac{\eta_{\infty}}{2}\right)^{2}\left[\sum_{j=0}^{N} d_{i, j}^{(1)} f\left(z_{j}\right)-\left(\frac{\eta_{\infty}}{2}\right) \lambda\right] \\
& +\left(\frac{\eta_{\infty}}{2}\right)^{3} \lambda^{2}=0,
\end{aligned}
$$




$$
\begin{aligned}
& \left(\frac{1}{\operatorname{Pr}}+R\right) \sum_{j=0}^{N} d_{i, j}^{(2)} \theta\left(z_{j}\right)+f\left(z_{i}\right)\left(\frac{\eta_{\infty}}{2}\right) \sum_{j=0}^{N} d_{i, j}^{(1)} \theta\left(z_{j}\right) \\
& +\gamma\left(\frac{\eta_{\infty}}{2}\right)^{2} \theta\left(z_{i}\right)+\mathrm{Nb} \sum_{j=0}^{N} d_{i, j}^{(1)} \phi\left(z_{j}\right) \sum_{j=0}^{N} d_{i, j}^{(1)} \theta\left(z_{j}\right) \\
& +\mathrm{Nt}\left(\sum_{j=0}^{N} d_{i, j}^{(1)} \theta\left(z_{j}\right)\right)^{2}=0 \\
& \sum_{j=0}^{N} d_{i, j}^{(2)} \phi\left(z_{j}\right)+\operatorname{Le} f\left(z_{i}\right)\left(\frac{\eta_{\infty}}{2}\right) \sum_{j=0}^{N} d_{i, j}^{(1)} \phi\left(z_{j}\right) \\
& +\frac{\mathrm{Nt}}{\mathrm{Nb}} \sum_{j=0}^{N} d_{i, j}^{(2)} \theta\left(z_{j}\right)=0 . \\
& f\left(z_{N}\right)=S, \\
& \left(\frac{\eta_{\infty}}{2}\right)^{2} \sum_{j=0}^{N} d_{N, j}^{(1)} f\left(z_{j}\right)=\left(\frac{\eta_{\infty}}{2}\right)^{3} \\
& +\delta_{1}\left(\frac{\eta_{\infty}}{2}\right) \sum_{j=0}^{N} d_{N, j}^{(2)} f\left(z_{j}\right)+\delta_{2} \sum_{j=0}^{N} d_{N, j}^{(3)} f\left(z_{j}\right), \\
& \theta\left(z_{N}\right)=1, \\
& \phi\left(z_{N}\right)=1 \text {, } \\
& \sum_{j=0}^{N} d_{0, j}^{(1)} f\left(z_{j}\right)=\lambda, \\
& \theta\left(z_{0}\right)=0, \\
& \phi\left(z_{0}\right)=0 \text {. }
\end{aligned}
$$

The resulting nonlinear equations (20)-(22) are associated with the boundary conditions equations (23) and (24) that contain $2 \mathrm{~N}-1$ equations which are solved using Newton method. The computer program of the numerical method was executed in MATHEMATICA 9 running on a PC. For more details of transforming the domain of the problem $\left[0, \eta_{\infty}\right]$ to the Chebyshev one, that is, $[-1,1]$, definition of the associated collocation points $\left(z_{j}, j=0,1, \ldots, N\right)$ and the matrix entries $d_{i, j}^{(k)}$, where $k$ th is the derivative of any selected function, the reader is advised to see [35]. Recently, this approach has been applied in nano boundary layer study $([34,35])$, and further, Aly [40] and Aly and Sayed [41] have used it for investigating the nanofluids flow.

\section{Results and Discussion}

In this work, suction/injection and heat source/sink flow near a stagnation-point over a heated stretching sheet in a porous medium saturated by a nanofluid, with effect of thermal radiation and magnetic field in the presence of the velocity slip model, have been studied. As presented in Section 2, the governing system of partial differential equations has been transformed into a system of nonlinear ordinary equations using similarity transformations. It was found that the solution depends on magnetic $(M)$, permeability $(K)$, and radiation $(R)$ parameters; Prandtl $(\mathrm{Pr})$ and Lewis (Le) numbers; Brownian motion $(\mathrm{Nb})$ and thermophoresis $(\mathrm{Nt})$ coefficients; heat source/sink $(\gamma)$; suction/injection $(S)$; first-order $\left(\delta_{1}\right)$ and second-order $\left(\delta_{2}\right)$ slip factors. Then, the obtained system has been numerically solved by the Chebyshev pseudospectral differentiation matrix (ChPDM) as briefly introduced in Section 3.

It should be noted that the current velocity slip model affects directly equation of the stream function $f(\eta)$, as shown in the boundary condition (15), and therefore its inflow involves also for the temperature $\theta(\eta)$ and concentration $\phi(\eta)$ (volume fraction of nanoparticles). In the next subsection, the importance of applying this slip model on investigating system (12)-(14) is to be studied. For implementation of this model, comparison with already published results is done in some special cases for different changes of the selected parameters, where the dotted curves refer to the present results.

\subsection{Implementation of the Present Physical Model}

4.1.1. Special Case 1: When $K=R=\gamma=S=0$. Figure 1 illustrates influence of the velocity ratio $\lambda$ on the stream velocity profile $f^{\prime}(\eta)$ when $\operatorname{Pr}=1$, Le $=2, \mathrm{Nb}=\mathrm{Nt}=0.2$, $M=1, \delta_{1}=\delta_{2}=0$ (solid curves as in Figure 2 in [23]), and $\delta_{1}=1, \delta_{2}=-1$. This graph shows that when $\lambda>1(<1)$, the stream velocity increases (decreases) uniformly over the entire domain and the boundary layer thickness decreases as values of $\lambda$ increase. However, as the slip model presents, the influence of the stream velocity reaches very rapidly the stable manner at $\eta_{\infty}$, achieving the boundary condition at infinity for both cases of $\lambda$. This is because, as expected for the fluid flows at nanoscales, the shear stress at the wall decreases with an increase in the first- and second-order slip parameters (see $[10,34,35])$. Hence, the stream velocity becomes slower and, however, temperature and concentration (volume fraction of nanoparticles) increase, as shown in the next three figures.

In Figure 2, we plot effect of the magnetic field $M$ on the stream velocity profile $f^{\prime}(\eta)$ when $\operatorname{Pr}=1$, Le $=2, \mathrm{Nb}=\mathrm{Nt}=$ $0.2, \lambda=3.3, \delta_{1}=\delta_{2}=0$ (solid curves as in Figure 3 in [23]), and $\delta_{1}=0.1, \delta_{2}=-0.1$. This figure shows that the boundary layer thickness decreases as the values of $M$ increase. This matches the physical view on applying the magnetic field to an eclectically conducting fluid, and this gives a rise in the Lorentz force, which results in retarding force on the velocity; that is, it slows down the motion of the fluid. In presence of the slip present model, even for small values of $\delta_{1}=0.1$ and $\delta_{2}=-0.1$, value of the stream velocity $f^{\prime}(\eta)$ increases as compared with absence of the slip at the same value of $M$. Further, for large values of $\delta_{1}$ and $\left|\delta_{2}\right|$ and as explained for the previous figure, it is expected that the velocity profiles reach very quickly to $\eta_{\infty}$, achieving the boundary condition.

Figure 3 shows the temperature distribution $\theta \eta$ for various values of the velocity ratio $\lambda$ when $\mathrm{Pr}=\mathrm{Le}=1, \mathrm{Nb}=$ $\mathrm{Nt}=0.5, M=1, \delta_{1}=\delta_{2}=0$ (solid curves as Figure 6 in [23]), and $\delta_{1}=1, \delta_{2}=-1$. From this figure, it is seen that as 


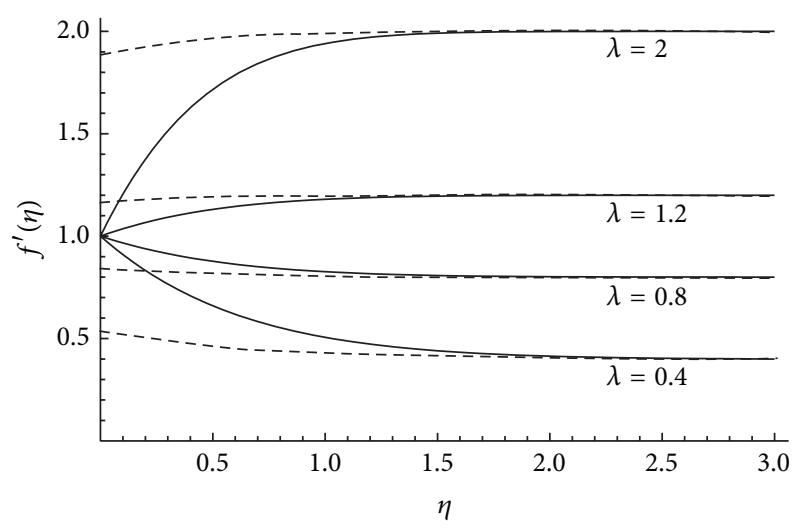

_ Ibrahim et al. [23]

- - Present (with $\delta_{1}=1, \delta_{2}=-1$ )

FIGURE 1: Effect of the velocity ratio $\lambda$ on the stream velocity profile $f^{\prime}(\eta)$ when $\operatorname{Pr}=1, \mathrm{Le}=2, \mathrm{Nb}=0.2, \mathrm{Nt}=0.2, M=1, K=R=\gamma=$ $S=0, \delta_{1}=\delta_{2}=0$ (solid curves as in Figure 2 in Ibrahim et al. [23]), and $\delta_{1}=1, \delta_{2}=-1$ (dotted curves as in the present).

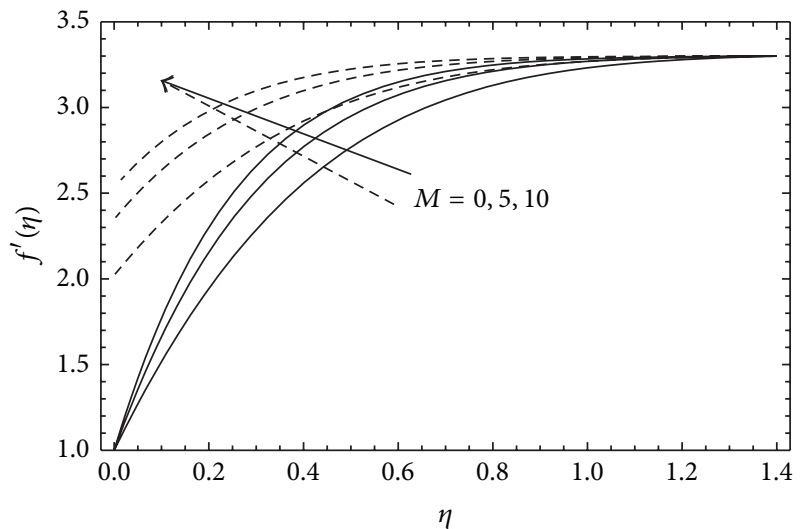

— Ibrahim et al. [23]

- - Present (with $\delta_{1}=0.1, \delta_{2}=-0.1$ )

Figure 2: Effect of the magnetic field $M$ on the stream velocity profile $f^{\prime}(\eta)$ when $\operatorname{Pr}=1, \mathrm{Le}=2, \mathrm{Nb}=0.2, \mathrm{Nt}=0.2, \lambda=3.3$, $K=R=\gamma=S=0, \delta_{1}=\delta_{2}=0$ (solid curves as in Figure 3 in Ibrahim et al. [23]), and $\delta_{1}=0.1, \delta_{2}=-0.1$ (dotted curves as in the present).

$\lambda$ increases the thermal boundary layer thickness decreases. It should be noted that when $\delta_{1} \neq 0$ and $\delta_{2} \neq 0$ and for fixed value of $\lambda$ the temperature boundary thickness increases and this result is very remarkable when $\lambda=0$; that is, the free stream velocity has the same value of the stretching velocity.

Figure 4 depicts the variation of temperature graph with respect to Prandtl number $\operatorname{Pr}$ when $\mathrm{Le}=1, \mathrm{Nb}=\mathrm{Nt}=0.5$, $M=1, \lambda=0.2, \delta_{1}=\delta_{2}=0$ (solid curves as Figure 7 in [23]), and $\delta_{1}=1, \delta_{2}=-1$. Due to the fact that a higher $\operatorname{Pr}$ in nanofluids study has relatively low thermal conductivity, which reduces conduction and thereby the thermal boundary layer thickness, Figure 4 shows that an increase of Prandtl

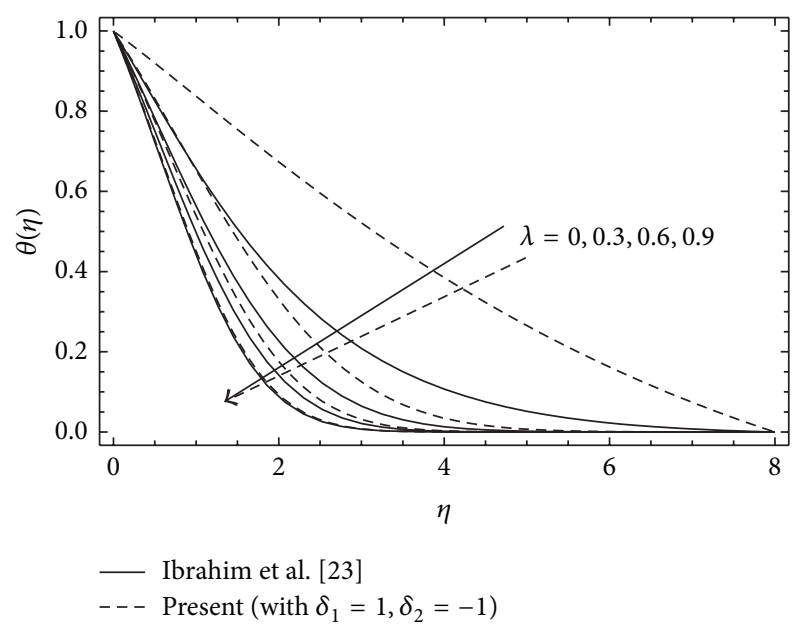

FIGURE 3: Effect of the velocity ratio $\lambda$ on the temperature distribution $\theta(\eta)$ when $\operatorname{Pr}=1$, Le $=1, \mathrm{Nb}=0.5$, Nt $=0.5, M=1$, $K=R=\gamma=S=0, \delta_{1}=\delta_{2}=0$ (solid curves as in Figure 6 in Ibrahim et al. [23]), and $\delta_{1}=1, \delta_{2}=-1$ (dotted curves as in the present).

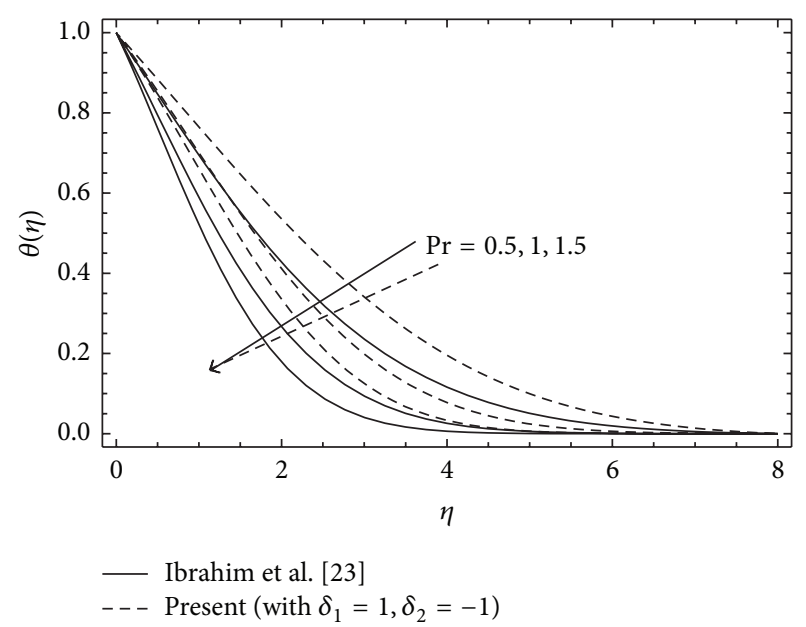

Figure 4: Effect of Prandtl number Pr on the temperature distribution $\theta(\eta)$ when $\mathrm{Le}=1, \mathrm{Nb}=0.5, \mathrm{Nt}=0.5, M=1, \lambda=0.2$, $K=R=\gamma=S=0, \delta_{1}=\delta_{2}=0$ (solid curves as in Figure 7 in Ibrahim et al. [23]), and $\delta_{1}=1, \delta_{2}=-1$ (dotted curves as in the present).

number leads to a decrease in the temperature profile. Again, on the presence of the slip model and for fixed value of Pr, the temperature boundary thickness increases.

Effect of Lewis number on the concentration distribution $\phi(\eta)$ when $\operatorname{Pr}=1, \mathrm{Nb}=\mathrm{Nt}=0.5, M=1, \lambda=0.2$, $\delta_{1}=\delta_{2}=0$ (solid curves as Figure 9 in [23]), and $\delta_{1}=1$, $\delta_{2}=-1$ is demonstrated in Figure 5. This figure reveals that the concentration boundary layer thickness decreases as Le increases, which is expected because a great of Lewis number increases the mass transfer rate; as a result increasing of Le decreases the concentration profiles. However, presence of the slip model increases the concentration boundary layer 


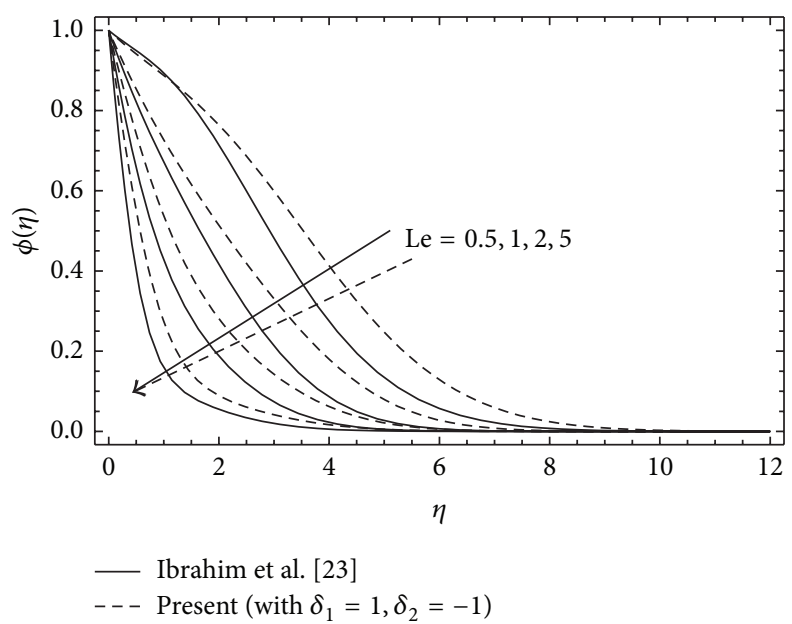

FIGURE 5: Effect of Lewis number Le on the concentration distribution $\phi(\eta)$ (volume fraction of nanoparticles) when $\mathrm{Pr}=1, \mathrm{Nb}=0.5$, $\mathrm{Nt}=0.5, M=1, \lambda=0.2, K=R=\gamma=S=0, \delta_{1}=\delta_{2}=0$ (solid curves as in Figure 9 in Ibrahim et al. [23]), and $\delta_{1}=1, \delta_{2}=-1$ (dotted curves as in the present).

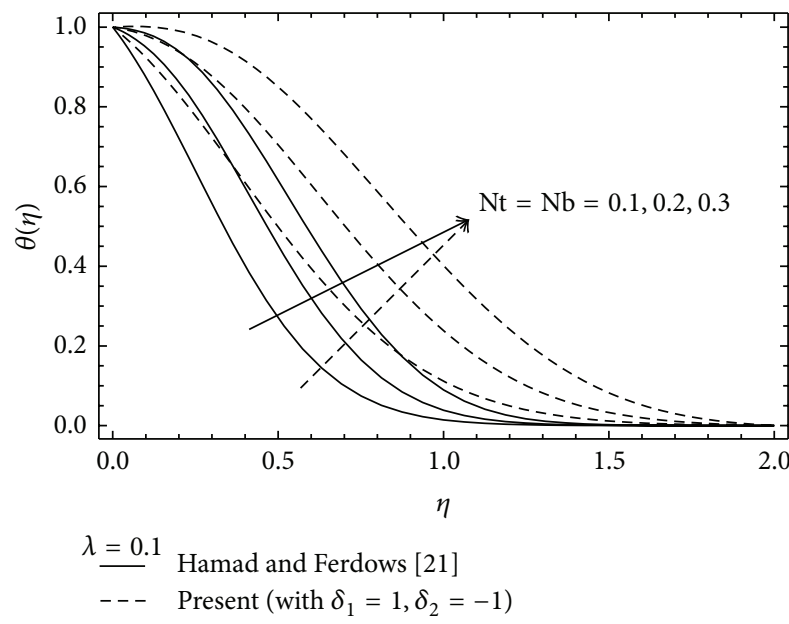

(a)

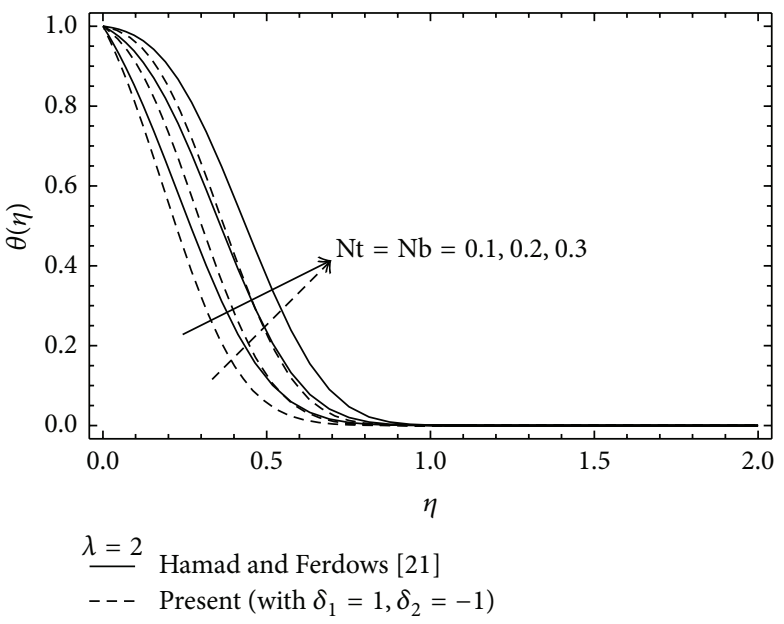

(b)

Figure 6: Effect of $\mathrm{Nt}$ and $\mathrm{Nb}$ on temperature distribution $\theta(\eta)$ when $\operatorname{Pr}=10$, Le $=10, S=0.1, \gamma=0.1, K=0.1, M=R=0, \delta_{1}=\delta_{2}=0$ (solid curves as in Figure 2 in Hamad and Ferdows [21]), and $\delta_{1}=1, \delta_{2}=-1$ (dotted curves as in the present) for (a) $\lambda=0.1(<1)$ and (b) $\lambda=2(>1)$.

thickness for fixed value of Lewis number. Further, the difference between the curves without and with the slip, at the same values of the other parameters, increases as the value of Le increases.

4.1.2. Special Case 2: When $M=R=0$. Figures 6(a) and 6(b) and $7(\mathrm{a})$ and $7(\mathrm{~b})$ represent variation of the temperature $\theta(\eta)$ and concentration $\phi(\eta)$ when $\operatorname{Pr}=\mathrm{Le}=10, S=\gamma=K=0.1$, $\delta_{1}=\delta_{2}=0$ (solid curves as in Figures 2 and 3 in [21]), and $\delta_{1}=1, \delta_{2}=-1$ for (a) $\lambda=0.1(<1)$, (b) $\lambda=2(>1)$ and two sets of values $\mathrm{Nt}=\mathrm{Nb}=0.1,0.2,0.3$ as in Figure 6, while $\mathrm{Nt}=0.1$ and $\mathrm{Nb}=0.1,0.2,0.3$ in Figure 7. In the absence of the slip model, Figure 6 shows that the temperature increases with the increase in $\mathrm{Nt}$ and $\mathrm{Nb}$ and the thermal boundary layer thickness for $\lambda<1$ is greater than for $\lambda>1$. However, in the presence of the slip model, this thickness becomes greater for $\lambda<1$ but smaller for $\lambda>1$. The same result can be also noticed in Figure 7. In addition, when $\delta_{1}=1$ and $\delta_{2}=-1$, the thickness of the boundary layer for the thermal boundary layer thickness is now remarkable; it is greater than the mass fraction. It should be mentioned here that this is the same result obtained in Section 4.1.1 for $\lambda<1$ but there is a vice versa behaviour for $\lambda>1$.

Effect of Lewis number in suction $(S>0)$ and injection $(S<0)$ cases on the temperature distribution for $\operatorname{Pr}=10$, $\mathrm{Nb}=\mathrm{Nt}=0.3, \lambda=2, \gamma=0.1, K=1, \delta_{1}=\delta_{2}=0$ (solid curves as in Figure 4 in [21]), $\delta_{1}=1$ and $\delta_{2}=-1$ is shown in Figure 8. It is seen that the thermal boundary layer 


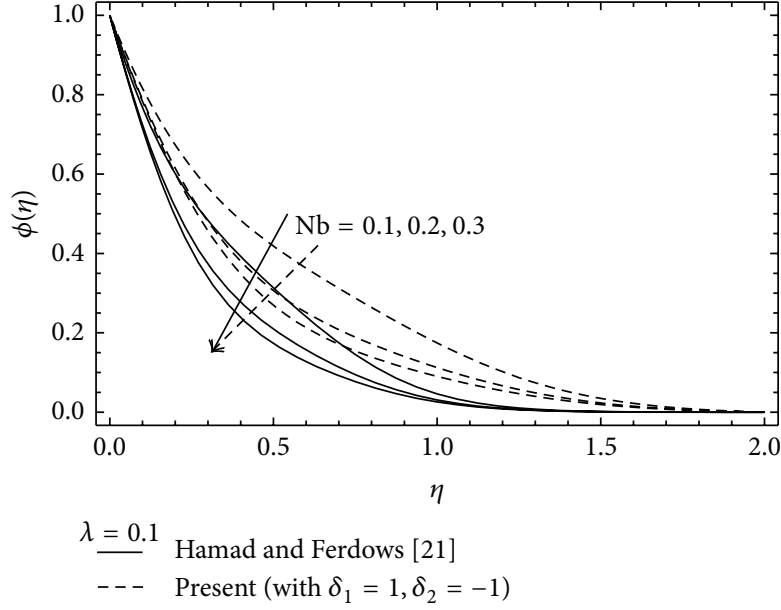

(a)

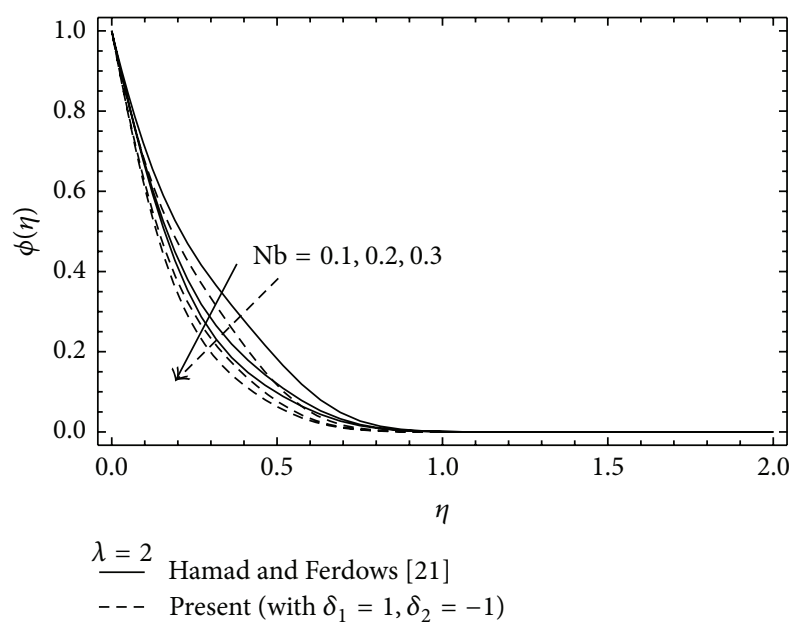

(b)

Figure 7: Effect of $\mathrm{Nb}$ on concentration distribution $\phi(\eta)$ (volume fraction of nanoparticles) when $\operatorname{Pr}=10, \mathrm{Le}=10, \mathrm{Nt}=0.1, S=0.1$, $\gamma=0.1, K=0.1, M=R=0, \delta_{1}=\delta_{2}=0$ (solid curves as in Figure 3 in Hamad and Ferdows [21]), and $\delta_{1}=1, \delta_{2}=-1$ (dotted curves as in the present) for (a) $S=1$ (suction) and (b) $\S=-1$ (injection).

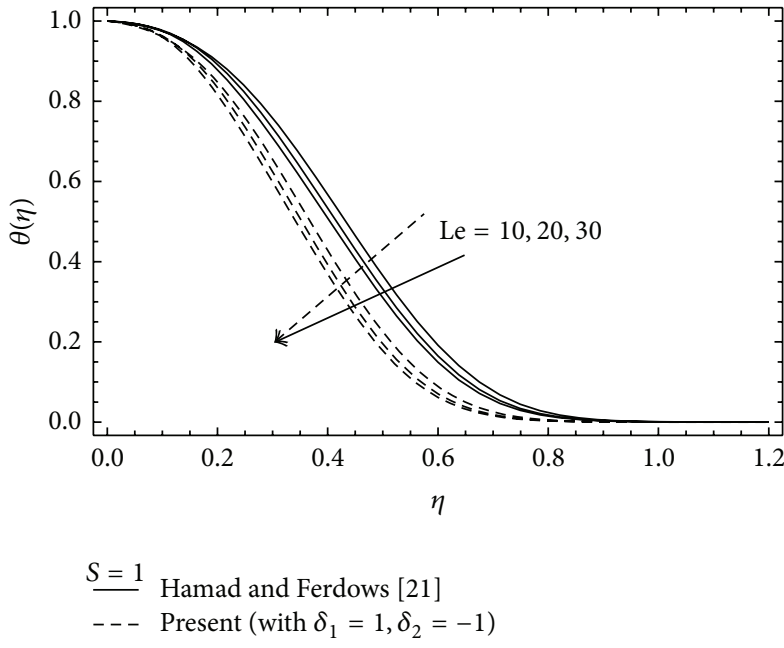

(a)

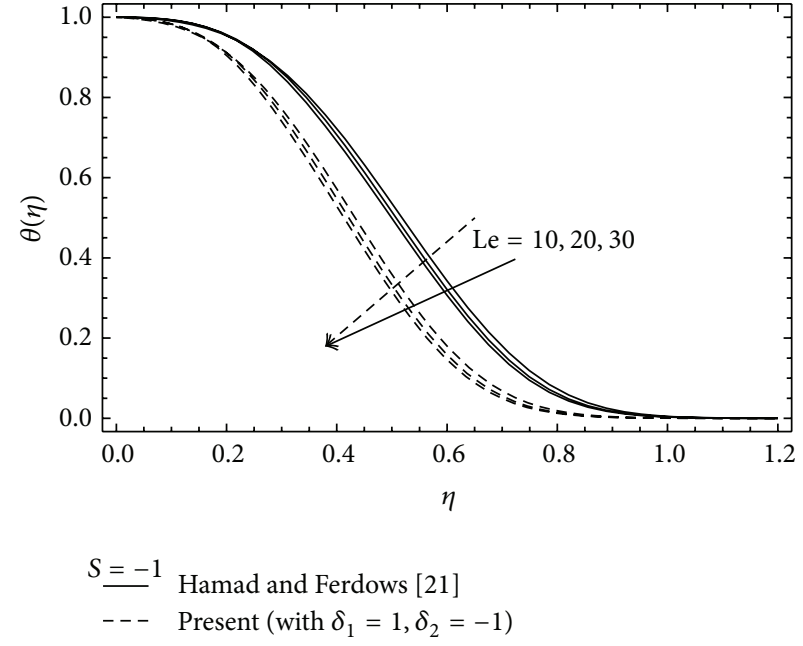

(b)

Figure 8: Effect of Lewis number Le temperature distribution $\theta(\eta)$ when $\operatorname{Pr}=10, \mathrm{Nb}=0.3, \mathrm{Nt}=0.3, \lambda=2, \gamma=0.1, K=1, M=R=0$, $\delta_{1}=\delta_{2}=0$ (solid curves as in Figure 4 in Hamad and Ferdows [21]), and $\delta_{1}=1, \delta_{2}=-1$ (dotted curves as in the present) for (a) $\lambda=0.1(<1)$ and (b) $\lambda=2(>1)$.

thicknesses decrease with increasing Le for both injection and suction cases, whilst for fixed value of Le, this thickness decreases in the presence of the slip model with a bigger decreasing in the injection case.

Figures 9(a) and 9(b) indicate variation of the temperature distribution with various values of the Prandtl number Pr when Le $=10, \mathrm{Nb}=0.3, \mathrm{Nt}=0.1, S=0.1, \lambda=2$, $K=1, \delta_{1}=\delta_{2}=0$ (solid curves as in Figure 6 in [21]), and $\delta_{1}=1, \delta_{2}=-1$ for (a) $\gamma=0.1>0$ (heat source) and (b) $\lambda=-0.1<0$ (sink). From this figure, one can deduce that, inside (outside) the thermal boundary layer, the value of temperature is high (low) with high value of Pr in both cases of the sink and heat source. In addition, for fixed value of Pr, this layer thickness becomes smaller on applying the present slip model. This result is absolutely different from that one obtained in discussing Figure 4 for the same parameter $\operatorname{Pr}$ but matches the effect of $\lambda>1$ in Figures 6(a) and 7(a) for different values of $\mathrm{Nb}$ and $\mathrm{Nt}$.

4.1.3. Special Case 3: When $M=\gamma=S=0$. Figure 10 presents the effect of $\lambda$ on the concentration distribution $\phi(\eta)$ when $\operatorname{Pr}=1, \mathrm{Le}=1, \mathrm{Nb}=0.2, \mathrm{Nt}=0.1, K=0.1, R=0.5$, 


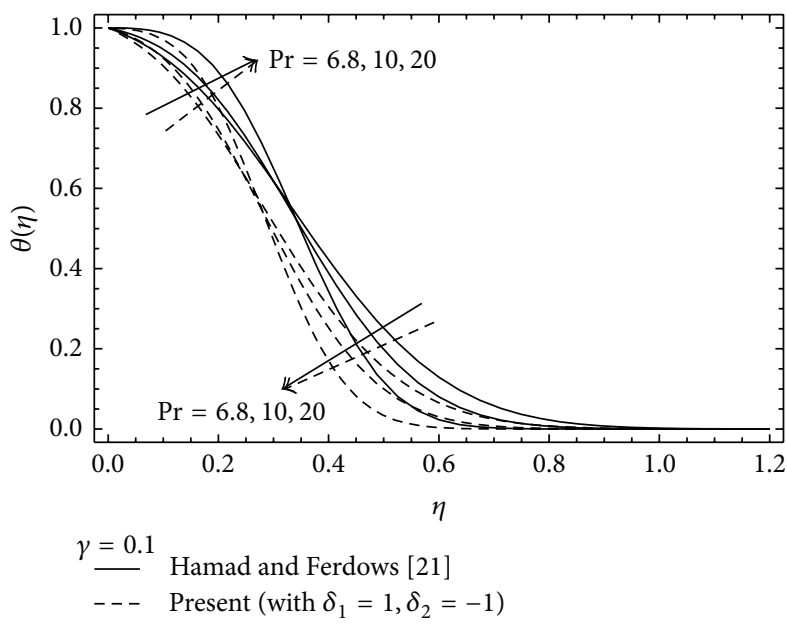

(a)

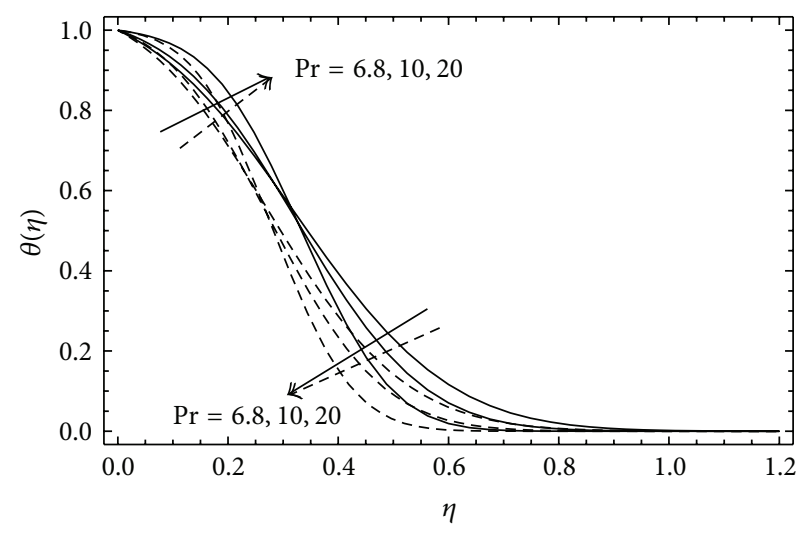

$\begin{array}{ll}\gamma=-0.1 & \text { Hamad and Ferdows [21] } \\ --- & \text { Present (with } \delta_{1}=1, \delta_{2}=-1 \text { ) }\end{array}$

(b)

FIGURE 9: Effect of $\operatorname{Pr}$ on concentration distribution $\phi(\eta)$ (volume fraction of nanoparticles) when Le $=10, \mathrm{Nb}=0.3, \mathrm{Nt}=0.1, S=0.1$, $\lambda=2, K=1, M=R=0, \delta_{1}=\delta_{2}=0$ (solid curves as in Figure 6 in Hamad and Ferdows [21]), and $\delta_{1}=1, \delta_{2}=-1$ (dotted curves as in the present) for (a) $\gamma=0.1>0$ (heat source) and (b) $\lambda=-0.1<0$ (sink).

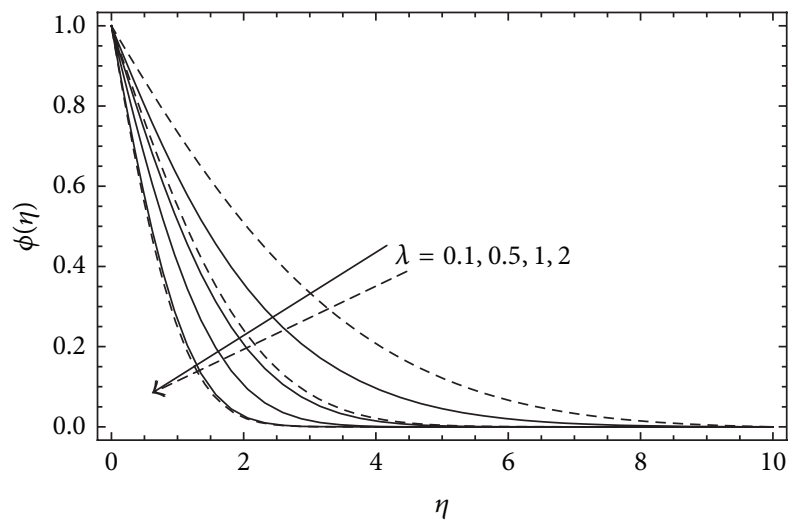

- Zheng et al. [22] (with dropping the temperature jump) --- Present (with $\delta_{2}=-10$ )

Figure 10: Effect of the velocity ratio $\lambda$ on the concentration distribution $\phi(\eta)$ (volume fraction of nanoparticles) when $\operatorname{Pr}=1$, $\mathrm{Le}=1, \mathrm{Nb}=0.2, \mathrm{Nt}=0.1, K=0.1, R=0.5, M=\gamma=S=0$, $\delta_{1}=0.5, \delta_{2}=0$ (solid curves as in Figure 11 in Zheng et al. [22] with dropping the temperature jump), and $\delta_{1}=0.5, \delta_{2}=-10$ (dotted curves as in the present).

$\delta_{1}=0.5, \delta_{2}=0$ (solid curves as in Figure 11 in [22] with dropping the temperature jump), and $\delta_{1}=0.5, \delta_{2}=-10$. This figure shows that an increase in $\lambda$ leads the concentration boundary layer thickness to decrease. However, it becomes greater (smaller) on applying the second slip factor $\delta_{2}$ for $\lambda<$ $1(\lambda<1)$. This result is compatible with that one obtained in Sections 4.1.1 and 4.1.2.

Important Remark. The results of this section demonstrate clearly that the full velocity slip model, that is, in the presence of the first-order and second-order slip factors, is necessary to predict the flow characteristics accurately. This agrees with the result obtained recently by Roşca and Pop [33], Aly and Ebaid [14], and Aly and Vajravelu [34]. Therefore, the full velocity slip model should be applied in the nanofluid mechanics, as shown in the next subsection.

4.2. Effect of the Slip Model on Full Length of the Problem. Besides the physical discussion of the velocity profile in Figure 1, Figures 11(a) and 11(b) show profiles of the velocity $f^{\prime}(\eta)$ and shear stress $f^{\prime \prime}(\eta)$ for variation of the first-order slip $\delta_{1}$ and second-order slip $\delta_{2}$ for two cases $\lambda=0.1<1$ and $\lambda=2>1$ when $S=-0.1, \operatorname{Pr}=\mathrm{Le}=10, \mathrm{Nt}=\mathrm{Nb}=0.1, M=$ $R=1$, and $K=\gamma=0.1$. From Figure 11(a), that is, when $\lambda=$ $0.1<1$, it can be seen that increasing the two slip parameters $\delta_{1}$ and $\left|\delta_{2}\right|$ leads to the decrease of the lateral velocity near the surface and its increase at the large distances. In addition, in the presence of these slip factors, the velocity is more decreasing rather than its value at fixed $\delta_{1}$ for various values of $\delta_{2}$. It should be noted that the decrease of $f^{\prime}(0)$ revealed the flow of fluid which comes from stretching of the sheet; hence any increasing in the two slip factors causes a decrease in the flow velocity profiles. However, vise versa behaviour is to be noticed in Figure 11(b), that is, when $\lambda=2>1$. In addition, as indicated in Figures 11(a) and 11(b), magnitude of the wall shear stress decreases with the increase of the two slip factors this is expected in the nanoscale investigations (see $[10,34,35])$. Furthermore, as stated before, the influence of the stream velocity and shear stress reaches very rapidly to the stable manner achieving the boundary condition at infinity for both cases of $\lambda$ in applying the slip model. It should be mentioned that the same behaviour has been obtained for the values of $S=0.1$ and $\gamma=-0.1$ in fixing the other values as in the current figure. 
TABLE 1: Comparison of results for the shear stress at the surface $f^{\prime \prime}(0)$ when $\operatorname{Pr}=1, M=K=R=\gamma=\mathrm{Nt}=S=\delta_{1}=\delta_{2}=0$, and Nb $\rightarrow 0$.

\begin{tabular}{lcccc}
\hline$\lambda$ & Present results & Ibrahim et al. [23] & Mahapatra and Gupta [16] & Hayat et al. [42] \\
\hline 0.01 & -0.998029 & -0.9980 & - & -0.99802 \\
0.1 & -0.969386 & -0.9694 & -0.9694 & -0.96938 \\
0.2 & -0.918107 & -0.9181 & -0.9181 & -0.91810 \\
0.5 & -0.667264 & -0.6673 & -0.6673 & -0.66732 \\
1.0 & 0.000000 & 0.0000 & 0.0000 & 0.00000 \\
2.0 & 2.017500 & 2.0175 & 2.0175 & 2.01750 \\
3.0 & 4.729280 & 4.7292 & 4.7293 & 4.72928 \\
\hline
\end{tabular}

TABLE 2: Comparison of results for the reduced Nusselt number $-\theta^{\prime}(0)$ when $M=K=R=\gamma=\mathrm{Nt}=S=\delta_{1}=\delta_{2}=0$ and $\mathrm{Nb} \rightarrow 0$.

\begin{tabular}{llcccc}
\hline $\operatorname{Pr}$ & $\lambda$ & \multicolumn{3}{c}{$-\theta^{\prime}(0)$} \\
\hline 1 & 0.1 & Present results & Ibrahim et al. [23] & Mahapatra and Gupta [16] & Hayat et al. [42] \\
\hline 1 & 0.2 & 0.624469 & 0.6022 & 0.603 & 0.602156 \\
1 & 0.5 & 0.692449 & 0.6245 & 0.625 & 0.624467 \\
\hline 1.5 & 0.1 & 0.776800 & 0.6924 & 0.692 & 0.692460 \\
1.5 & 0.2 & 0.797122 & 0.7768 & 0.777 & 0.776802 \\
1.5 & 0.5 & 0.864794 & 0.7971 & 0.797 & 0.797122 \\
& & Present results & Hamad and Ferdows [21] & 0.863 & 0.864771 \\
\hline 0.7 & 0 & 0.454181 & 0.45391 & Wang [43] & 0.4539 \\
2 & 0 & 0.911356 & 0.91136 & 0.9114 & 0.4539 \\
7 & 0 & 1.895360 & 1.89540 & 1.8954 & 0.9113 \\
\hline
\end{tabular}

Distribution of the temperature $\theta(\eta)$ and concentration $\phi(\eta)$ are plotted in Figures 12(a), and 12(b), 13(a), and 13(b), respectively, for variation of the two slip parameters $\delta_{1}$ and $\delta_{2}$ when $S=-0.1, \operatorname{Pr}=\mathrm{Le}=10, \mathrm{Nt}=\mathrm{Nb}=0.1$, $M=R=1, K=\gamma=0.1$, and $\lambda=0.1$. These figures show that, as expected, thickness of the temperature and concentration boundary layers are smaller than thickness of the velocity boundary layer in Figure 11. In addition, as $\delta_{1}$ and $\left|\delta_{2}\right|$ increase, the temperature of flow field and concentration of the nanoparticles increase for the investigated parameters. In addition, increasing the second-order slip parameter increases the boundary layer thickness of the temperature and concentration.

Quantities of practical interest, namely, the shear stress at the surface $\left[f^{\prime \prime}(0)\right]$, reduced Nusselt number $\left[\right.$ Nur $\left.=-\theta^{\prime}(0)\right]$, and reduced Sherwood number $\left[\mathrm{Shr}=-\phi^{\prime}(0)\right]$ are to be investigated in the next few figures. Further, a comparison of the present results with literature values shown in Tables 1 , 2 , and 3 for $f^{\prime \prime}(0),-\theta^{\prime}(0)$ and $-\phi^{\prime}(0)$, respectively, indicates very good agreement and hence a trust in obtaining highly accurate results at the coming cases.

Figures 14(a)-14(e) present variation of the shear stress at the surface $f^{\prime \prime}(0)$ as a function of $M, K, R, S$, and $\lambda$ for different values of the second slip factor $\delta_{2}$ when $\operatorname{Pr}=$ Le $=$ $10, \mathrm{Nt}=\mathrm{Nb}=0.1, \gamma=0.1$, and $\delta_{1}=1$. As seen in Figures 14(a)-14(d), $f^{\prime \prime}(0)$ decreases remarkably in the increase of $\left|\delta_{2}\right|$. Further, Figure 14(e) indicates that the shear stress at
TABLE 3: Comparison of results for the reduced Sherwood number $-\phi^{\prime}(0)$ when $\operatorname{Pr}=\mathrm{Le}=10, M=K=R=\lambda=\gamma=S=\delta_{1}=\delta_{2}=0$.

\begin{tabular}{ccccc}
\hline $\mathrm{Nt}$ & $\mathrm{Nb}$ & Present results & Zheng et al. [22] & Khan and Pop [28] \\
\hline 0.1 & 0.1 & 2.12938 & 2.12939 & 2.1294 \\
0.2 & 0.1 & 2.27401 & 2.27402 & 2.2740 \\
0.3 & 0.1 & 2.52868 & 2.52863 & 2.5286 \\
0.4 & 0.1 & 2.79519 & 2.79517 & 2.7952 \\
0.5 & 0.1 & 3.03510 & 3.03514 & 3.0351 \\
0.1 & 0.2 & 2.38182 & 2.38187 & 2.3819 \\
0.1 & 0.3 & 2.40995 & 2.41001 & 2.4100 \\
0.1 & 0.4 & 2.39961 & 2.39965 & 2.3997 \\
0.1 & 0.5 & 2.38256 & 2.38257 & 2.3836 \\
\hline
\end{tabular}

the wall increases (decreases) with the increase of $\left|\delta_{2}\right|$ for $\lambda<1(\lambda>1)$. In addition, when $\lambda=1, f^{\prime \prime}(0)=0$ and this is because the sheet and the fluid move with the same velocity. However, at this case, they are at different temperature, that is, $-\theta^{\prime}(0) \neq 0$, and in concentration difference, that is, $-\phi^{\prime}(0) \neq$ 0 . This was examined and it was found that, when $\lambda=1$, $-\theta^{\prime}(0)=0.721082$ and $-\phi^{\prime}(0)=3.06155$.

Figures 15 and 16 show variation of the reduced Nusselt number (Nur) and reduced Sherwood number (Shr) as a function of the magnetic field parameter $M$ for parametric 


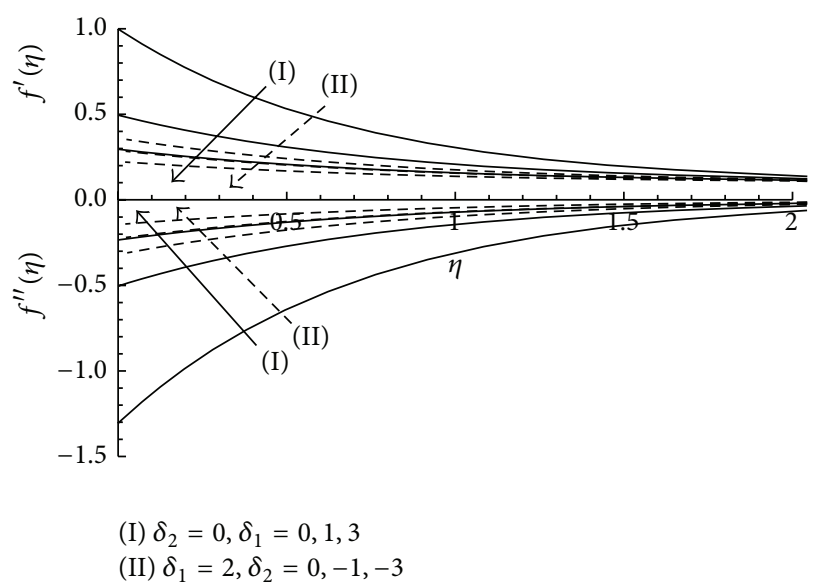

(a)

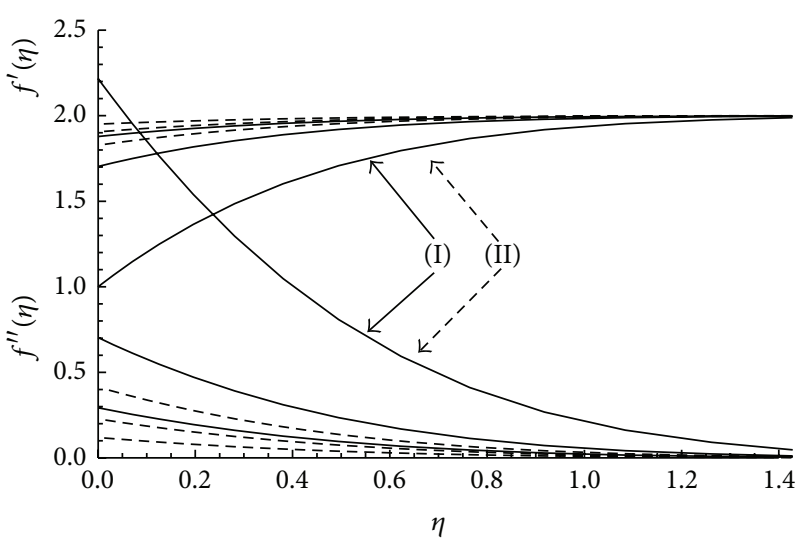

(I) $\delta_{2}=0, \delta_{1}=0,1,3$

(II) $\delta_{1}=2, \delta_{2}=0,-1,-3$

(b)

Figure 11: Profiles of $f^{\prime}(\eta)$ and $f^{\prime \prime}(\eta)$ for $S=-0.1, \operatorname{Pr}=\mathrm{Le}=10, \mathrm{Nt}=\mathrm{Nb}=0.1, M=R=1$, and $K=\gamma=0.1$, when (a) $\lambda=0.1$ and (b) $\lambda=2$.

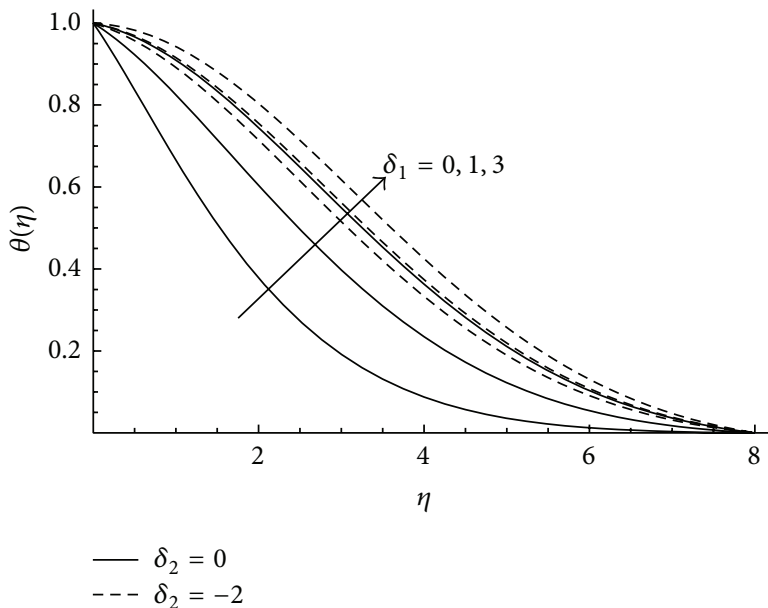

(a)

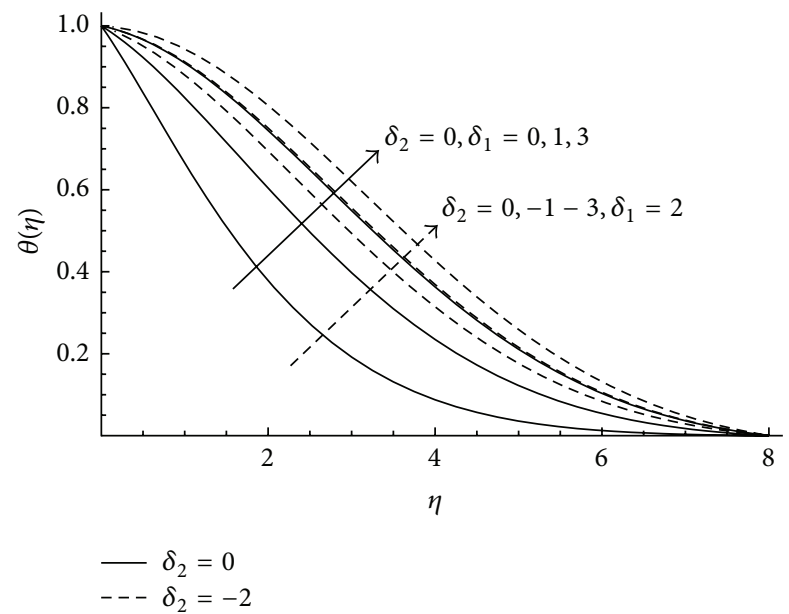

(b)

FIGURE 12: Effect of $\delta_{1}$ and $\delta_{2}$ on temperature distribution $\theta(\eta)$ for $S=-0.1$, Pr $=\mathrm{Le}=10, \mathrm{Nt}=\mathrm{Nb}=0.1, M=R=1, K=\gamma=0.1$, and $\lambda=0.1$, when (a) $\delta_{1}=0,1,3$ for $\delta_{2}=0$ (solid lines) and $\delta_{2}=-2$ (dotted lines) and (b) $\delta_{2}=0, \delta_{1}=0,1,3$ (solid lines) and $\lambda=2, \delta_{2}=0,-1,-3$ (dotted lines).

values of $\mathrm{Nt}=\mathrm{Nb}(=0.1,0.2,0.3)$, case $(\mathrm{a})$, Lewis number $(\mathrm{Le}=10,20,30)$, case $(\mathrm{b})$, and Prandtl number $(\operatorname{Pr}=$ $1,5,10)$, case $(\mathrm{c})$, in three influence parameters, namely, suction/injection $S\left(= \pm 0.1, \jmath_{g_{1}}\right.$ vt. $\jmath_{g_{2}}$, see the red ellipses), velocity ratio $\lambda$ ( $\gtrless 1, J_{g_{1}}$ vt. $\jmath_{g_{3}}$, notice the blue ellipses), and heat source $/ \operatorname{sink} \gamma\left(= \pm 0.1, \jmath_{g_{1}}\right.$ vt. $\jmath_{g_{4}}$, note the green ellipses $)$, where $\rho$ refers to the cases (a), (b), and (c). In these figures, direction of the arrow's head refers to the increase of the three influence parameters. In the rest of this section, the results are presented in symbols pattern, which may be more easier than sentences, especially in discussing huge number of parameters as in the current study. Hence, at the same value of $\mathrm{Nt}=\mathrm{Nb}$, Le, or Pr, the main results of these figures can be summarized as follows:

(1) For the reduced Nusselt number

(a) $\left(\left.\operatorname{Nur}_{\downarrow}\right|_{a_{g_{4} \uparrow}} ^{M}\right)>\left(\left.\operatorname{Nur}_{\downarrow}\right|_{a_{g_{1} \uparrow}} ^{M}\right)>\left(\left.\operatorname{Nur}_{\downarrow}\right|_{a_{g_{2} \uparrow} \uparrow} ^{M}\right)>$ $\left(\left.\operatorname{Nur}_{\downarrow}\right|_{a_{g_{3} \uparrow}} ^{M}\right)$

(b) $\left(\left.\operatorname{Nur}_{\cong}\right|_{b_{g_{4} \uparrow}} ^{M}\right)>\left(\left.\operatorname{Nur}_{\cong}\right|_{b_{g_{1} \uparrow}} ^{M}\right)>\left(\left.\operatorname{Nur}_{\cong}\right|_{b_{g_{2} \uparrow}} ^{M}\right)>$ $\left(\left.\operatorname{Nur}_{\approx}\right|_{b_{g_{3} \uparrow} \uparrow} ^{M}\right)$, where the difference is slightly small for all four cases of Le, 


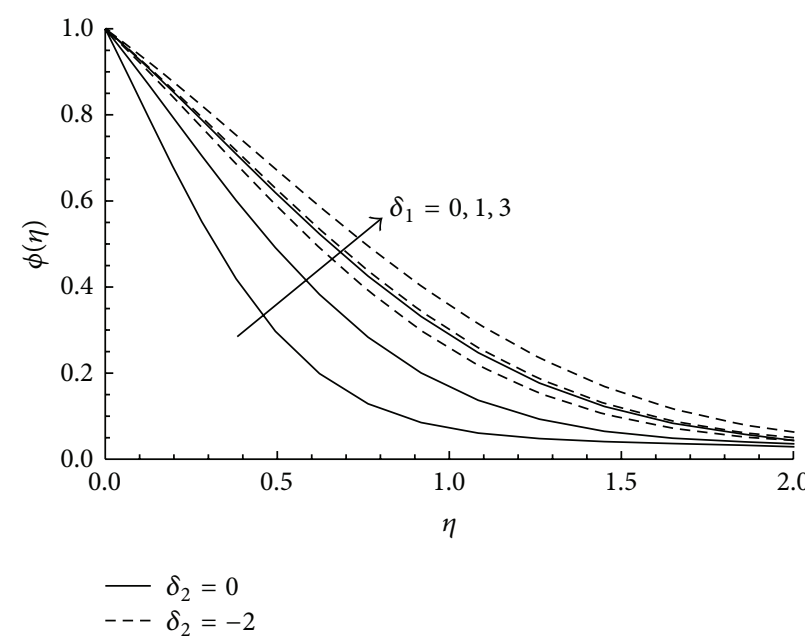

(a)

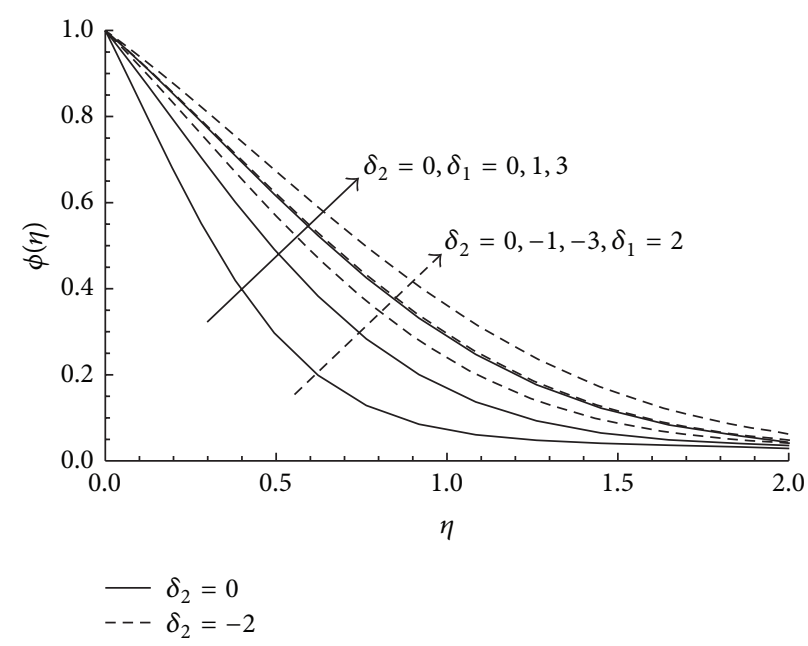

(b)

Figure 13: Effect of $\delta_{1}$ and $\delta_{2}$ on concentration distribution $\phi(\eta)$ for $S=-0.1, \operatorname{Pr}=\mathrm{Le}=10, \mathrm{Nt}=\mathrm{Nb}=0.1, M=R=1, K=\gamma=0.1$, and $\lambda=0.1$, when (a) $\delta_{1}=0,1,3$ for $\delta_{2}=0$ (solid lines) and $\delta_{2}=-2$ (dotted lines) and (b) $\delta_{2}=0, \delta_{1}=0,1,3$ (solid lines) and $\lambda=2, \delta_{2}=0,-1,-3$ (dotted lines).

(c) $\left(\left.\operatorname{Nur}_{\uparrow}\right|_{c_{g_{4} \uparrow}} ^{M}\right)>\left(\left.\operatorname{Nur}_{\uparrow}\right|_{c_{g_{1} \uparrow}} ^{M}\right)>\left(\left.\operatorname{Nur}_{\uparrow}\right|_{c_{g_{2} \uparrow}} ^{M}\right)>$ $\left(\left.\operatorname{Nur}{ }_{\cong}\right|_{c_{g_{3} \uparrow}} ^{M}\right)$, where the difference is slightly small when $\gamma=-0.1$. It is noticed here that increasing Pr is to increase the heat transfer rate at the surface because the temperature gradient at the surface increases.

(2) For the reduced Sherwood number
(a) $\left(\left.\operatorname{Shr}_{\uparrow}\right|_{a_{g_{1} \uparrow}} ^{M}\right)>\left(\left.\operatorname{Shr}_{\uparrow}\right|_{a_{g_{2} \uparrow} \uparrow} ^{M}\right), \quad\left(\left.\operatorname{Shr}_{\uparrow}\right|_{a_{g_{1} \uparrow}} ^{M}\right)>$ $\left(\left.\operatorname{Shr}_{\uparrow}\right|_{a_{g_{3} \uparrow}} ^{M}\right),\left(\left.\operatorname{Shr}_{\uparrow}\right|_{a_{g_{1} \uparrow}} ^{M}\right)>\left(\left.\operatorname{Shr}_{\uparrow}\right|_{a_{g_{4} \uparrow}} ^{M}\right)$,
(b) $\left(\left.\operatorname{Shr}_{\uparrow}\right|_{b_{g_{1} \uparrow}} ^{M}\right)>\left(\left.\operatorname{Shr}_{\uparrow}\right|_{b_{g_{2} \uparrow}} ^{M}\right),\left(\left.\operatorname{Shr}_{\uparrow}\right|_{b_{g_{1} \uparrow}} ^{M}\right)>\left(\left.\operatorname{Shr}_{\uparrow}\right|_{b_{g_{3} \uparrow}} ^{M}\right)$, $\left(\left.\operatorname{Shr}_{\uparrow}\right|_{b_{g_{1} \uparrow}} ^{M}\right) \approx\left(\left.\operatorname{Shr}_{\uparrow}\right|_{b_{g_{4} \uparrow}^{R}} ^{R}\right)$,
(c) $\left(\left.\operatorname{Shr}_{\downarrow}\right|_{c_{g_{1} \uparrow}} ^{M}\right)>\left(\left.\operatorname{Shr}_{\downarrow}\right|_{c_{g_{2} \uparrow}} ^{M}\right),\left(\left.\operatorname{Shr}_{\downarrow}\right|_{c_{g_{1} \uparrow}} ^{M}\right)>\left(\left.\operatorname{Shr}_{\approx}\right|_{c_{g_{3} \uparrow}} ^{M}\right)$, $\left(\left.\mathrm{Shr}_{\downarrow}\right|_{c_{g_{1} \uparrow}} ^{M}\right)>\left(\left.\mathrm{Shr}_{\downarrow}\right|_{c_{g_{4} \uparrow}} ^{M}\right)$, where he difference is very small when $\gamma=-0.1$.

Here, it should be mentioned that the behaviour of reduced Nusselt number and reduced Sherwood number for the permeability parameter $K$ has been examined and it was found as exactly the same of magnetic field parameter $M$, that has been discussed in Figures 15 and 16. This result is expected as shown in the structure of (12) for $M$ and $K$ parameters and can be also noticed in Figures 14(a) and 14(b). In addition, this result matches the results obtained by Ibrahim et al. [23] for investigating the magnetic parameter only in comparison with the results introduced by Hamad and Ferdows [21] for studying the permeability parameter only, when $\gamma=S=0$ (see, e.g., Figures 7 and 8 in [21] and Figures 12 and 14 in [23]).

Figures 17 and 18 depict variation of the reduced Nusselt number $\left[\right.$ Nur $=-\theta^{\prime}(0)$ ] and reduced Sherwood number
[Shr $\left.=-\phi^{\prime}(0)\right]$ as a function of the radiation parameter $R$ for the same values of the parameters and cases as in Figures 15 and 16. At the fixed value of $\mathrm{Nt}=\mathrm{Nb}$, Le, or $\mathrm{Pr}$, the results of these figures can be deduced as follows:

(1) For the reduced Nusselt number

(a) $\left(\left.\operatorname{Nur}_{\downarrow}\right|_{a_{g_{4} \uparrow} \uparrow} ^{R}\right)>\left(\left.\operatorname{Nur}_{\downarrow}\right|_{a_{g_{1} \uparrow}} ^{R}\right)>\left(\left.\operatorname{Nur}_{\downarrow}\right|_{a_{g_{2} \uparrow}} ^{R}\right)>$ $\left(\left.\operatorname{Nur}_{\downarrow}\right|_{a_{g_{3} \uparrow}} ^{R}\right)$,

(b) $\left(\left.\operatorname{Nur}_{\cong}\right|_{b_{g_{4}} \uparrow} ^{R}\right)>\left(\left.\operatorname{Nur}_{\approx}\right|_{b_{g_{1}} \uparrow} ^{R}\right)>\left(\left.\operatorname{Nur}_{\approx}\right|_{b_{g_{2} \uparrow}} ^{R}\right)>$ $\left(\left.\mathrm{Nur}_{\approx}\right|_{b_{g_{3} \uparrow}} ^{R}\right)$, where the difference is extremely small for all four cases of Le,

(c) $\left(\left.\operatorname{Nur}_{\uparrow}\right|_{c_{g_{4} \uparrow}} ^{R}\right)>\left(\left.\operatorname{Nur}_{\uparrow}\right|_{c_{g_{1} \uparrow}} ^{R}\right)>\left(\left.\operatorname{Nur}_{\uparrow}\right|_{c_{g_{2} \uparrow}} ^{R}\right)>$ $\left(\left.\operatorname{Nur}_{\uparrow}\right|_{c_{g_{3} \uparrow}} ^{R}\right)$, where the difference is very small when $\gamma=-0.1$ at $R>1$.

(d) In addition, the influence of Nur moves very rapidly in the region $[0,1]$, which may be taken as the most effective region in studying the radiation, and then the graphs for every group go uniformly to a close equal value.

(2) For the reduced Sherwood number

(a) $\left(\left.\operatorname{Shr}_{\uparrow}\right|_{a_{g_{1} \uparrow}} ^{R}\right)>\left(\left.\operatorname{Shr}_{\uparrow}\right|_{a_{g_{2} \uparrow}} ^{R}\right), \quad\left(\left.\operatorname{Shr}_{\uparrow}\right|_{a_{g_{1} \uparrow}} ^{R}\right)>$ $\left(\left.\operatorname{Shr} r_{\uparrow}\right|_{a_{g_{3} \uparrow}} ^{R}\right), \quad\left(\left.\operatorname{Shr}_{\uparrow}\right|_{a_{g_{1} \uparrow}} ^{R}\right)>\left(\left.\operatorname{Shr}_{\uparrow}\right|_{a_{g_{4} \uparrow}} ^{R}\right)$, with effective influence in the region $[0,1]$,

(b) $\left(\left.\operatorname{Shr}_{\uparrow}\right|_{b_{g_{1} \uparrow}} ^{R}\right)>\left(\left.\operatorname{Shr}_{\uparrow}\right|_{b_{g_{2} \uparrow}} ^{R}\right),\left(\left.\operatorname{Shr}_{\uparrow}\right|_{b_{g_{1} \uparrow}} ^{R}\right)>\left(\left.\operatorname{Shr}_{\uparrow}\right|_{b_{g_{3} \uparrow}} ^{R}\right)$, $\left(\left.\operatorname{Shr}_{\uparrow}\right|_{b_{g_{1} \uparrow}} ^{R}\right) \approx\left(\left.\operatorname{Shr}_{\uparrow}\right|_{b_{g_{4} \uparrow}} ^{R}\right)$,

(c) $\left(\left.\operatorname{Shr}_{\downarrow}\right|_{c_{g_{1} \uparrow}} ^{R}\right)>\left(\left.\operatorname{Shr}_{\downarrow}\right|_{c_{g_{2} \uparrow}} ^{R}\right),\left(\left.\operatorname{Shr}_{\downarrow}\right|_{c_{g_{1} \uparrow}} ^{R}\right)>\left(\left.\operatorname{Shr}_{\approx}\right|_{c_{g_{3}} \uparrow} ^{R}\right)$, $\left(\left.\mathrm{Shr}_{\downarrow}\right|_{c_{g_{1} \uparrow}} ^{R}\right)>\left(\left.\mathrm{Shr}_{\downarrow}\right|_{c_{g_{4} \uparrow}} ^{R}\right)$, with a very effective performance in the region $[0,1]$. 


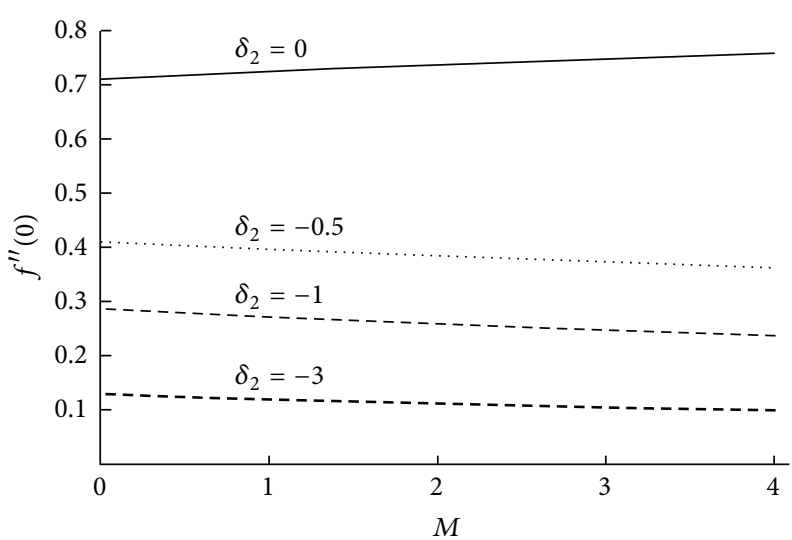

(a)

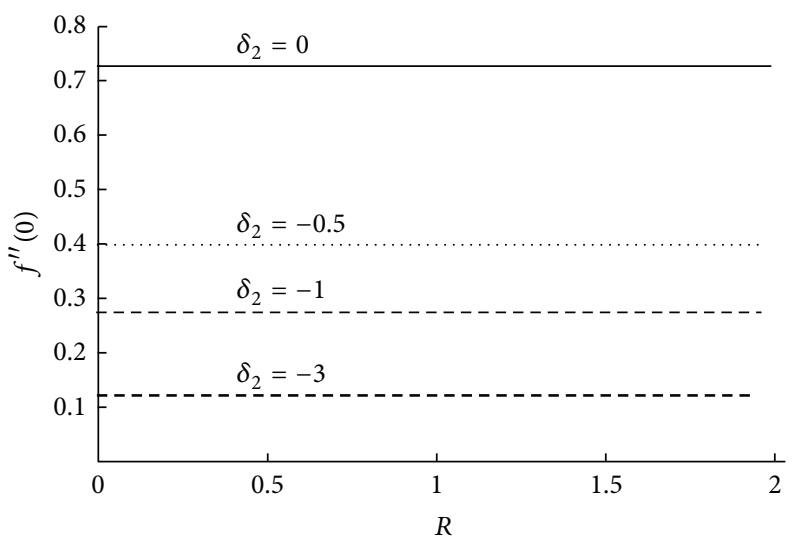

(c)

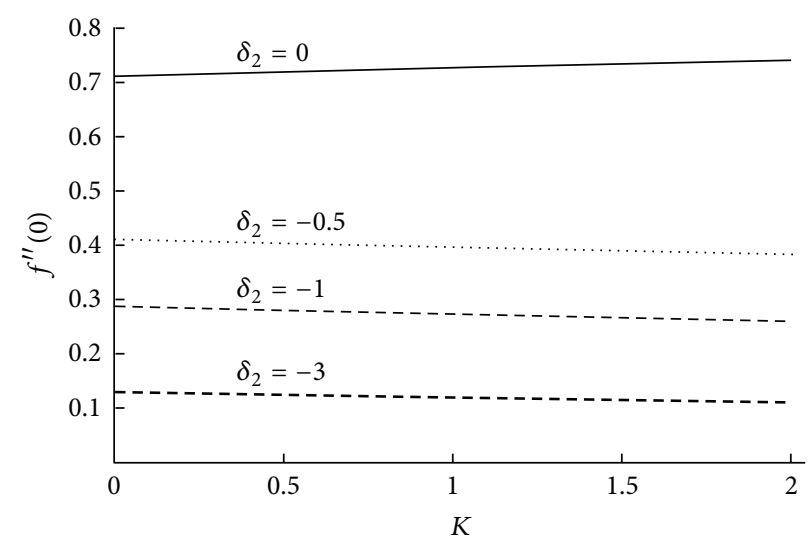

(b)

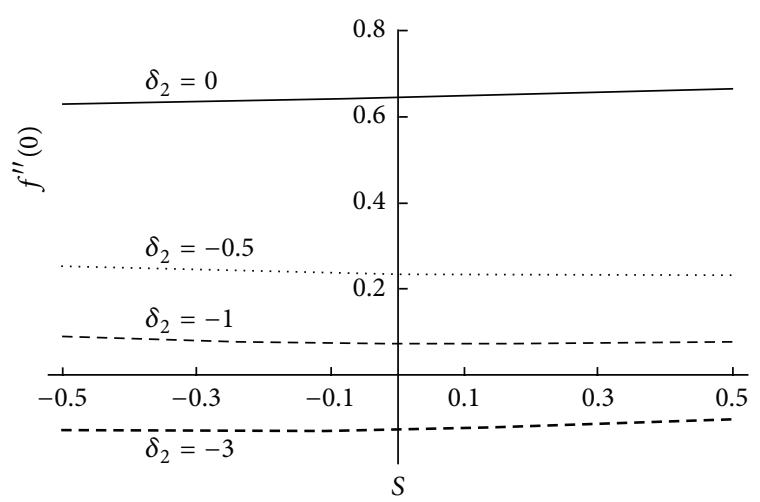

(d)

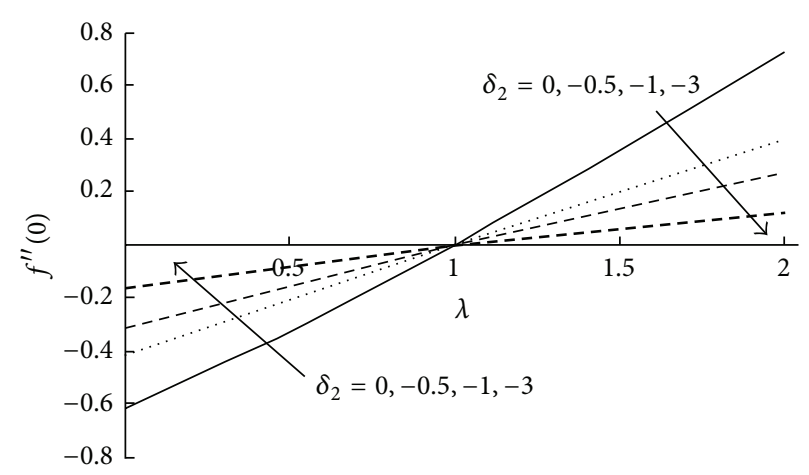

(e)

FIGURE 14: Effects of $\delta_{2}$ on relation of $f^{\prime \prime}(0)$ as a function of (a) $M$, (b) $K$, (c) $R$, (d) $S$, and (e) $\lambda$, where $M=1, K=1, R=1, S=0.1$, and $\lambda=2$ (if the parameter is not functionally in the above cases) and values of the other parameters are $\operatorname{Pr}=\mathrm{Le}=10, \mathrm{Nt}=\mathrm{Nb}=0.1, \gamma=0.1$, and $\delta_{1}=1$.

\section{Conclusion}

Boundary layer stagnation-point flow towards a heated stretching sheet in a porous medium saturated by a nanofluid in suction/injection cases and in the presence of radiation, magnetic field, and heat generation/absorption with effect of the full velocity slip boundary condition has been numerically solved. It was deduced that the solution of the main three functions, the stream $f(\eta)$, temperature $\theta(\eta)$, and concentration $\phi(\eta)$, depends on the following coefficients: magnetic field $(M)$, permeability $(K)$, radiation $(R)$, Prandtl number (Pr), Lewis number (Le), Brownian motion (Nb), thermophoresis $(\mathrm{Nt})$, heat source/sink $(\gamma)$, suction/injection $(S)$, first-order $\left(\delta_{1}\right)$ and second-order $\left(\delta_{2}\right)$ velocity slips. All of these parameters have been investigated besides the wall shear stress $f^{\prime \prime}(0)$, reduced Nusselt number $-\theta^{\prime}(0)$, and 

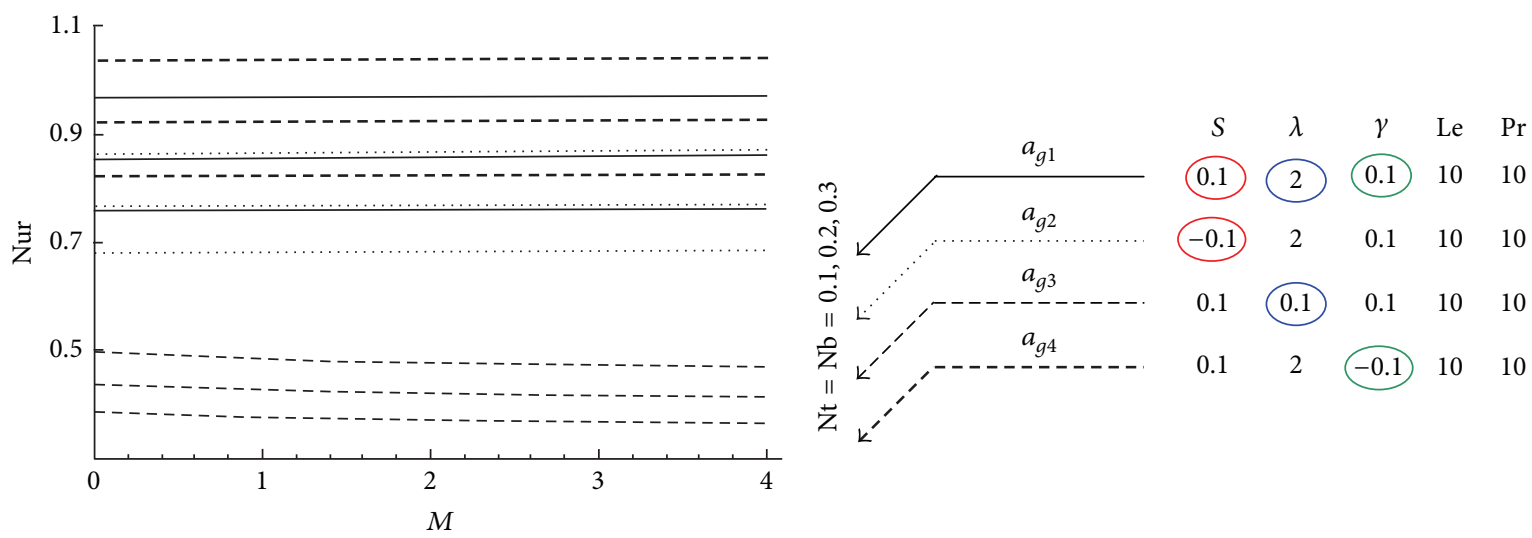

(a)
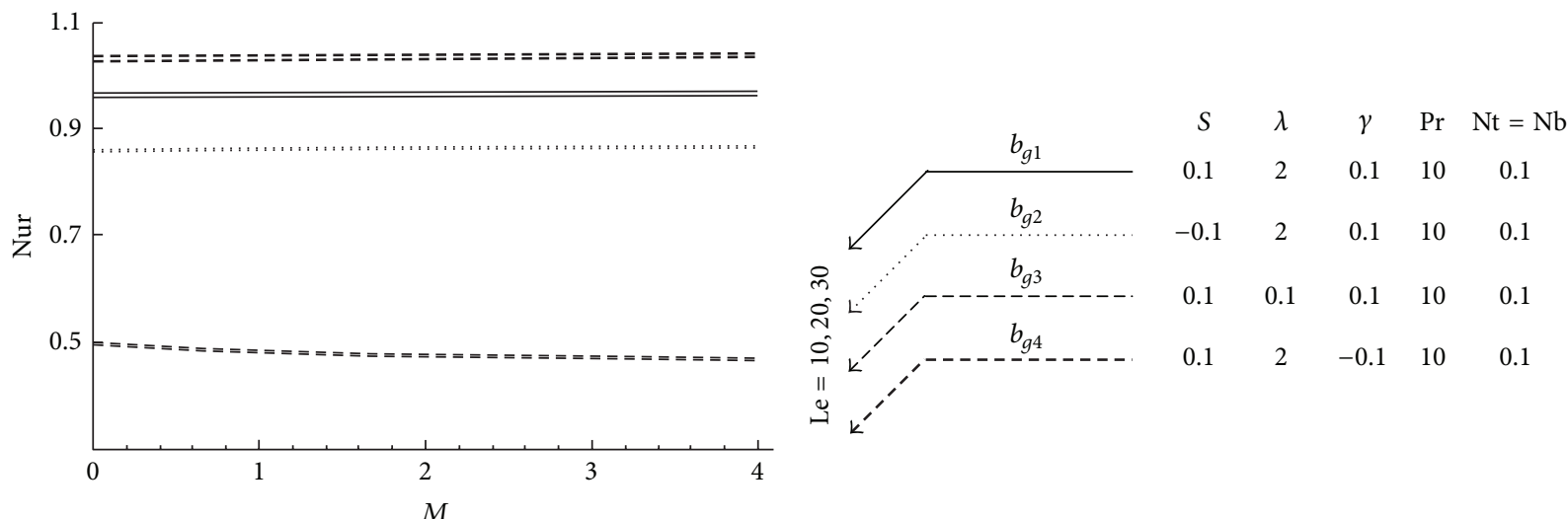

(b)
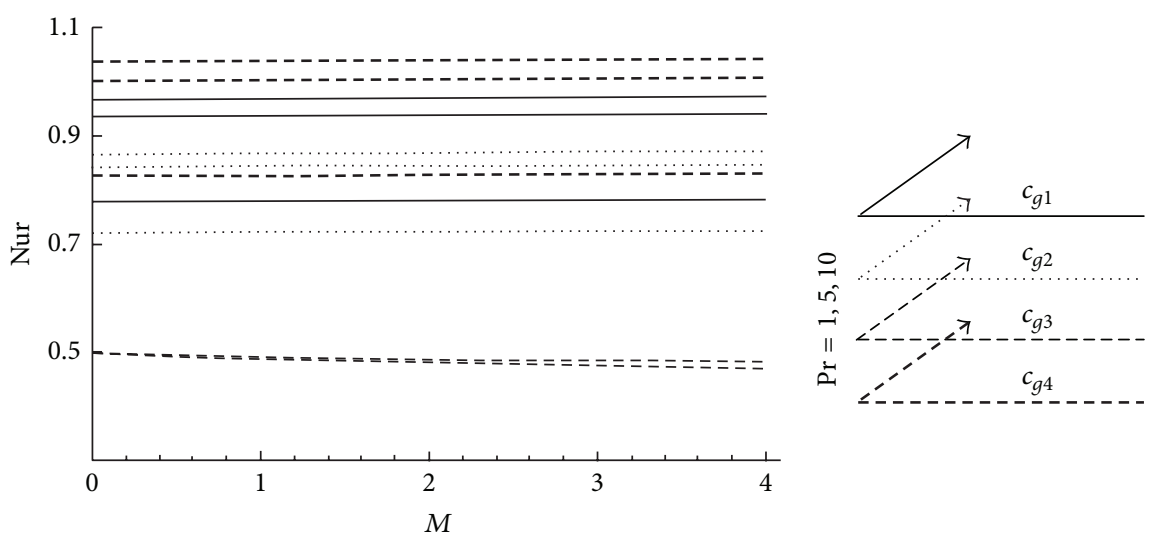

$$
\begin{array}{ccccc}
S & \lambda & \gamma & \mathrm{Le} & \mathrm{Nt}=\mathrm{Nb} \\
0.1 & 2 & 0.1 & 10 & 0.1 \\
-0.1 & 2 & 0.1 & 10 & 0.1 \\
0.1 & 0.1 & 0.1 & 10 & 0.1 \\
0.1 & 2 & -0.1 & 10 & 0.1
\end{array}
$$

(c)

Figure 15: Effects of Nt, Nb, Le, and Pr on relation of reduced Nusselt number [Nur $=-\theta^{\prime}(0)$ ] as a function of the magnetic parameter $M$ for $K=R=1, \delta_{1}=1, \delta_{2}=-1$, and $\gamma=0.1$.

reduced Sherwood number $-\phi^{\prime}(0)$. The main results of the present study can be epitomized as follows.

(1) For special cases, the current results are in a very good agreement with the results presented in $[16,21,23,28$, $42,43]$. Hence, the current results present the general case for those obtained in these references.

(2) It was shown that the flow velocity, surface shear stress, temperature, and concentration are strongly influenced on applying the full velocity slip. Therefore, for predicting the flow characteristics accurately, using this model is extremely important in the nanofluid mechanics.

(3) The influence of the stream velocity and shear stress reach very rapidly the stable manner for both cases of $\lambda$.

(4) The wall shear stress $f^{\prime \prime}(0)$ decreases (increases) remarkably in the increase of $\left|\delta_{2}\right|$ for $M, K, R, S$, and 

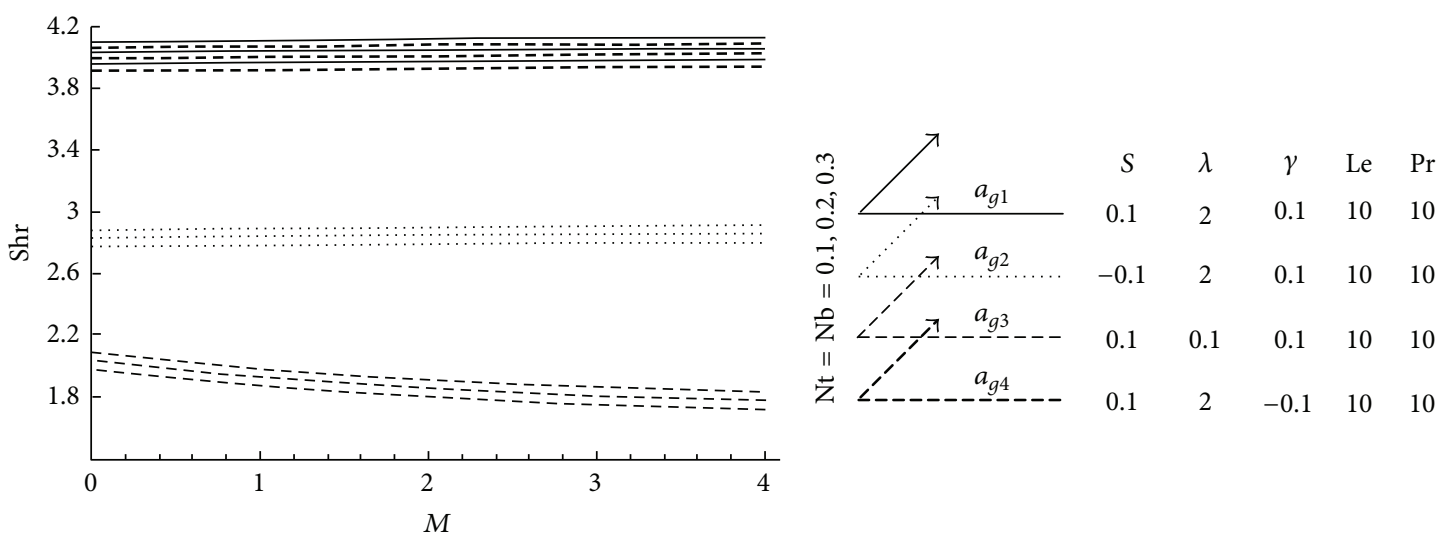

(a)
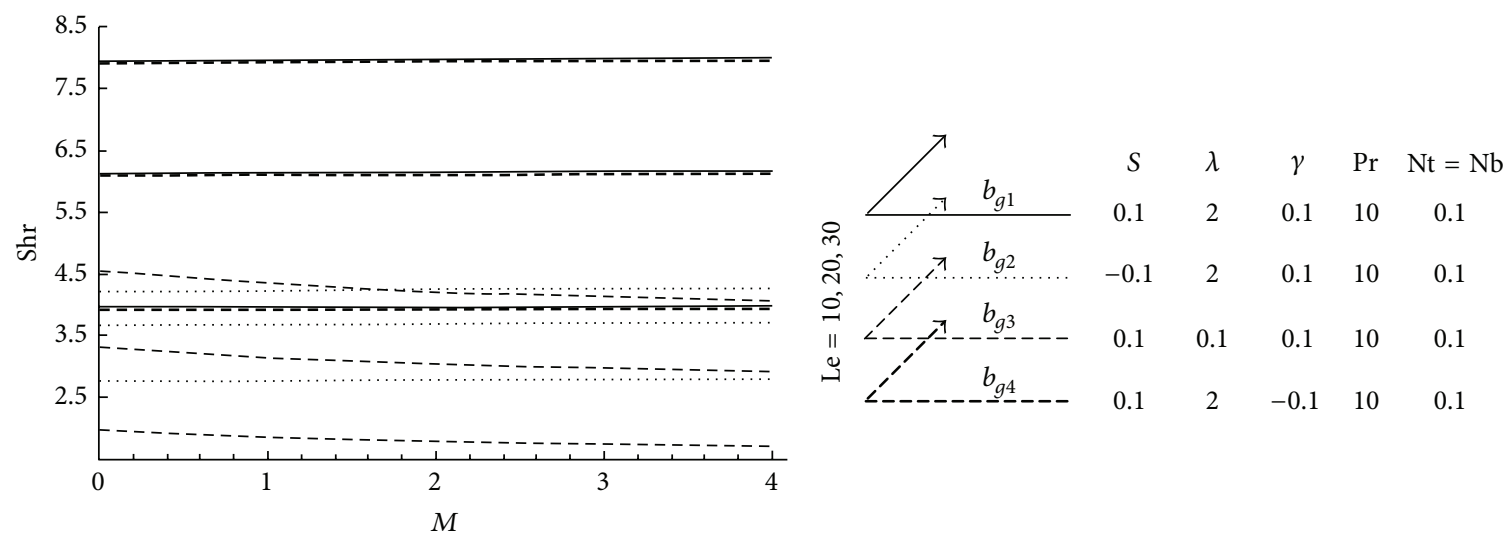

(b)
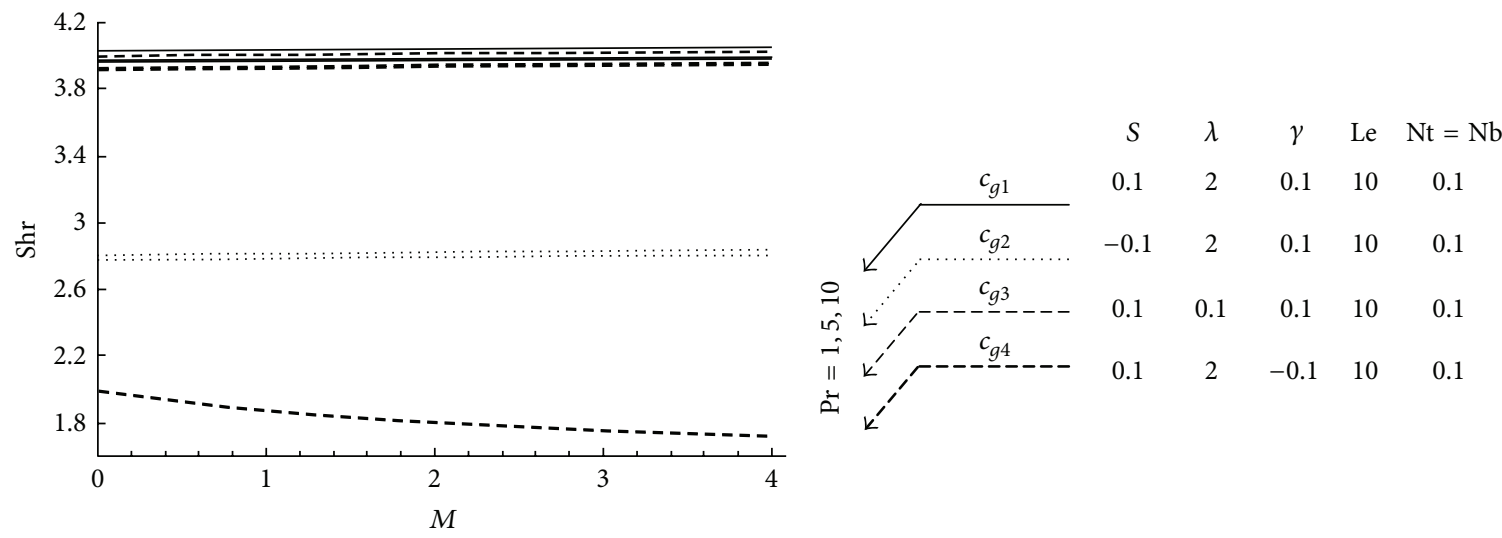

(c)

Figure 16: Effects of Nt, Nb, Le, and Pr on relation of reduced Sherwood number [Shr $=-\phi^{\prime}(0)$ ] as a function of the magnetic parameter $M$ for $K=R=1, \delta_{1}=1, \delta_{2}=-1$, and $\gamma=0.1$.

$\lambda>1(\lambda<1)$. When $\lambda=1$, the skin friction coefficient, reduced Nusselt number, and reduced Sherwood number all are constant. In particular, although $f^{\prime \prime}(0)=0$, however, $-\theta^{\prime}(0)=0.721082$ and $-\phi^{\prime}(0)=3.06155$.

(5) In presence of the magnetic field, permeability, and radiation and for the three investigated cases, namely, $\pm S, \lambda \gtrless 1$, and $\pm \gamma$, we have the following. (a) The reduced Nusselt number

(i) decreases with increase of $\mathrm{Nt}$ and $\mathrm{Nb}$,

(ii) has a very weak effect on increasing Le,

(iii) increases with increase of Pr,

(iv) is higher in the cases of suction, $\lambda>1$, and heat source in comparison with injection, $\lambda<1$, and heat sink, respectively. 

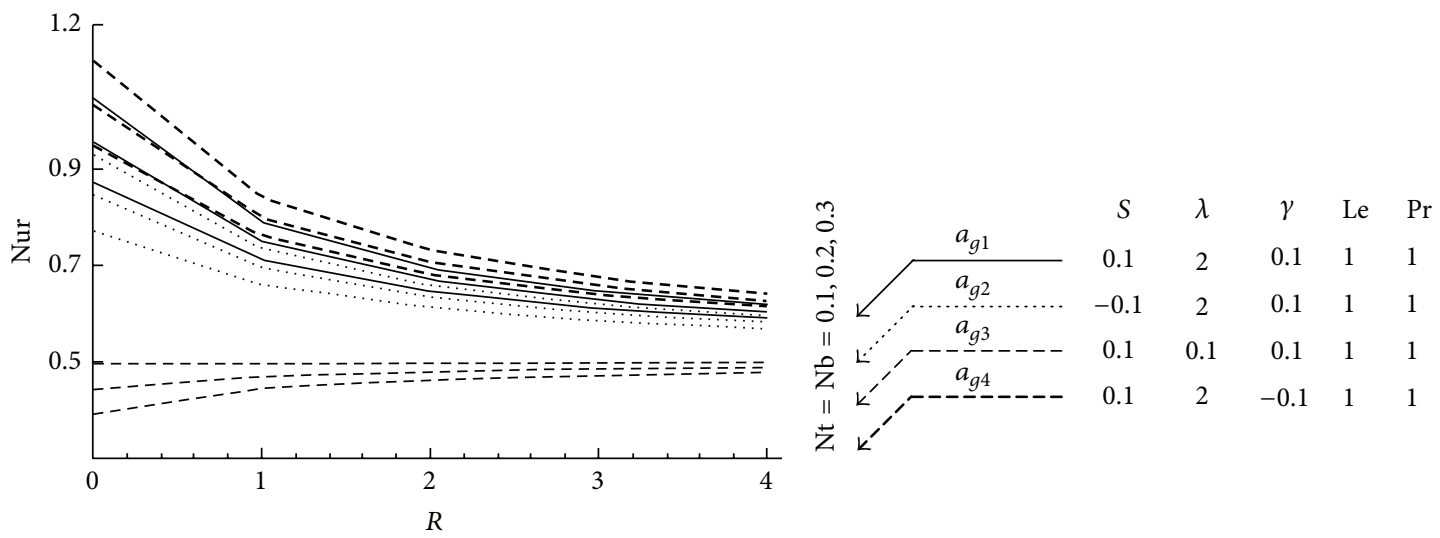

(a)
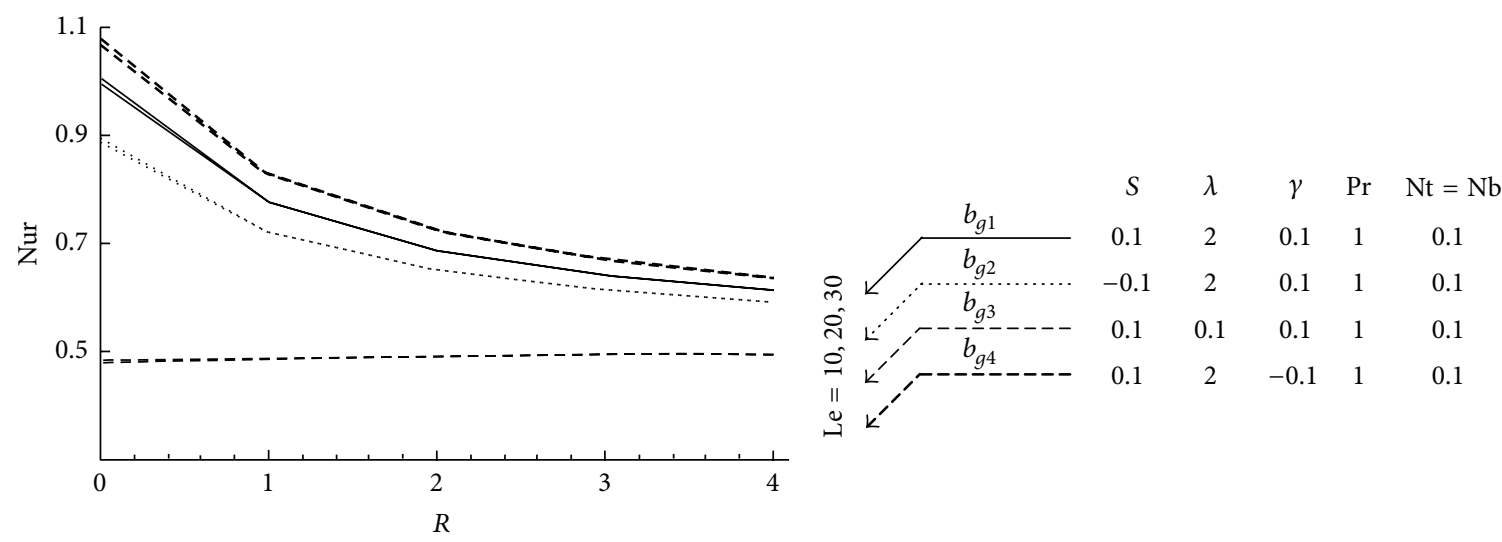

(b)
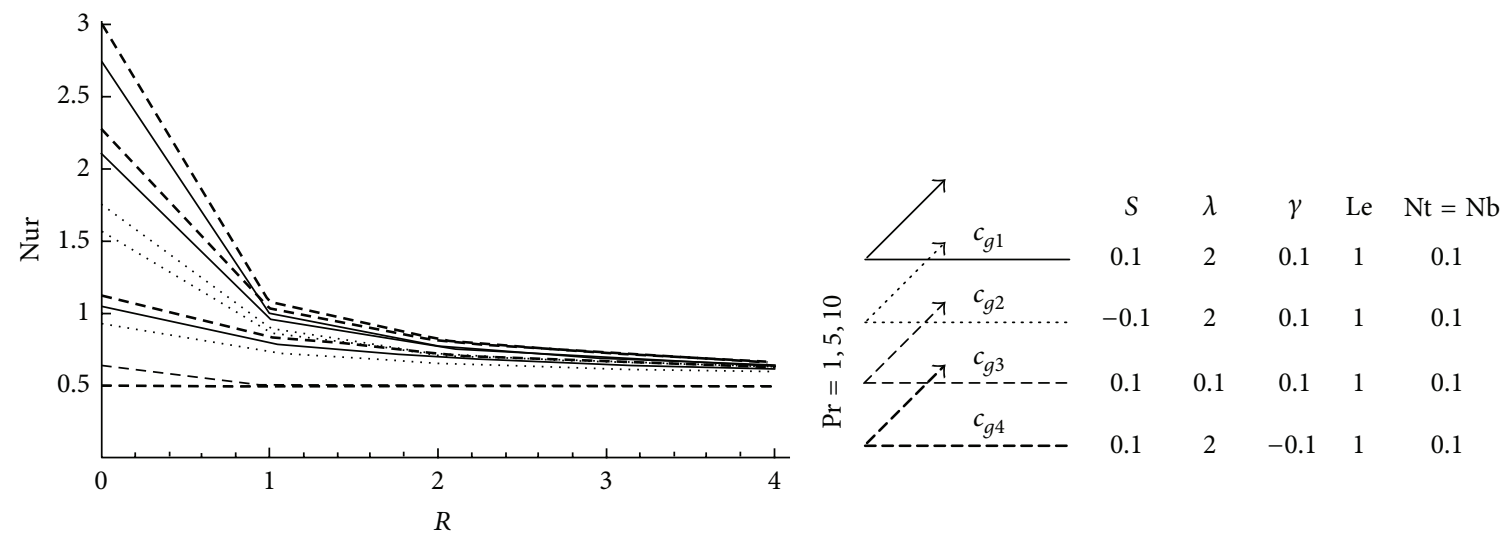

(c)

FIGURE 17: Effects of Nt, Nb, Le, and Pr on relation of reduced Nusselt number [Nur $=-\theta^{\prime}(0)$ ] as a function of the radiation parameter $R$ for $M=K=1, \delta_{1}=1, \delta_{2}=-1$, and $\gamma=0.1$.

(b) The reduced Sherwood number

(i) increases with increase of $\mathrm{Nt}, \mathrm{Nb}$, and Le,

(ii) decreases with increase of $\mathrm{Pr}$,

(iii) is higher in the cases of suction and $\lambda>1$ in comparison with injection and $\lambda<1$, respectively,

(iv) is approximately equal in the heat source and heat sink cases.
(6) The most effective region for radiation effect is $[0,1]$ in studying Nur and Shr.

Finally, one of the important results of the present research is that the velocity slip boundary condition of nanofluids is mandatory and should be taken into account in nanoscale research; otherwise a spurious physical sight is to be gained. This is because it has a very considerable and remarkable effect on the nanofluids' flow. 

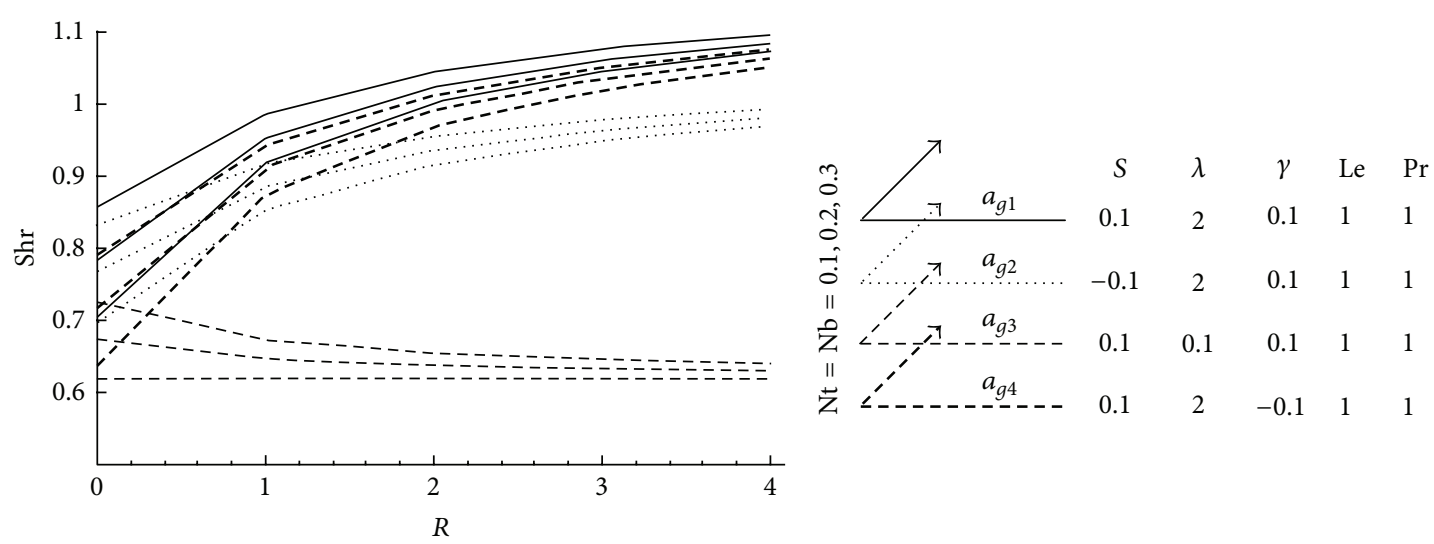

(a)
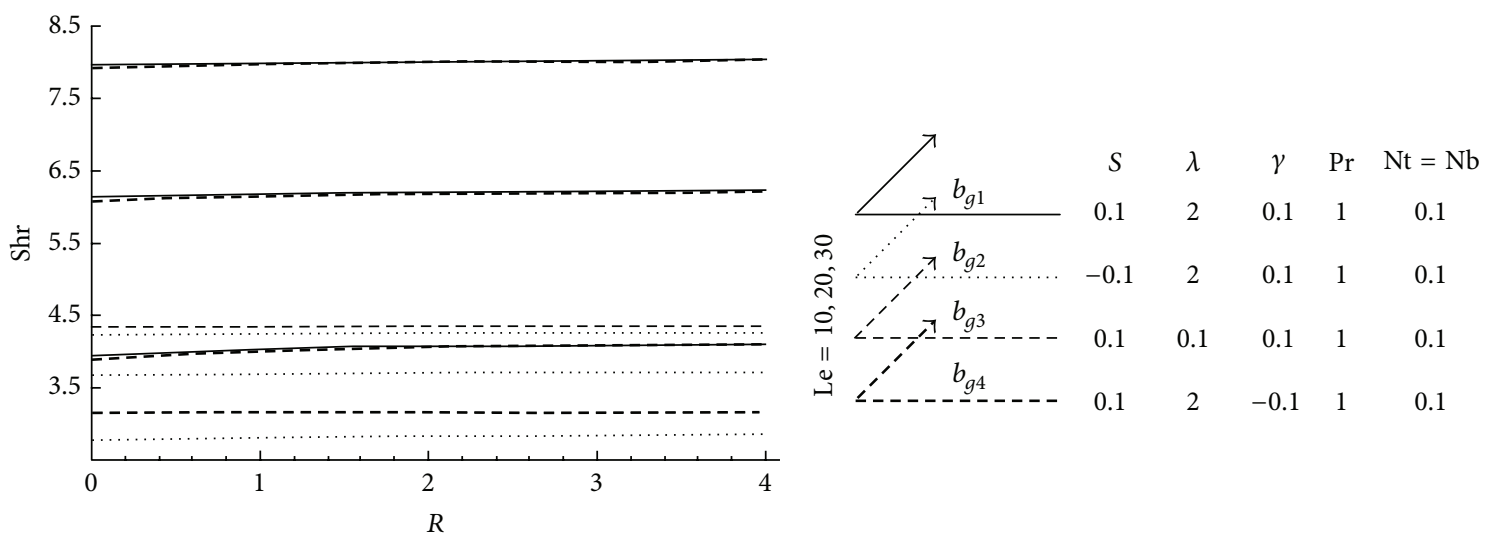

(b)
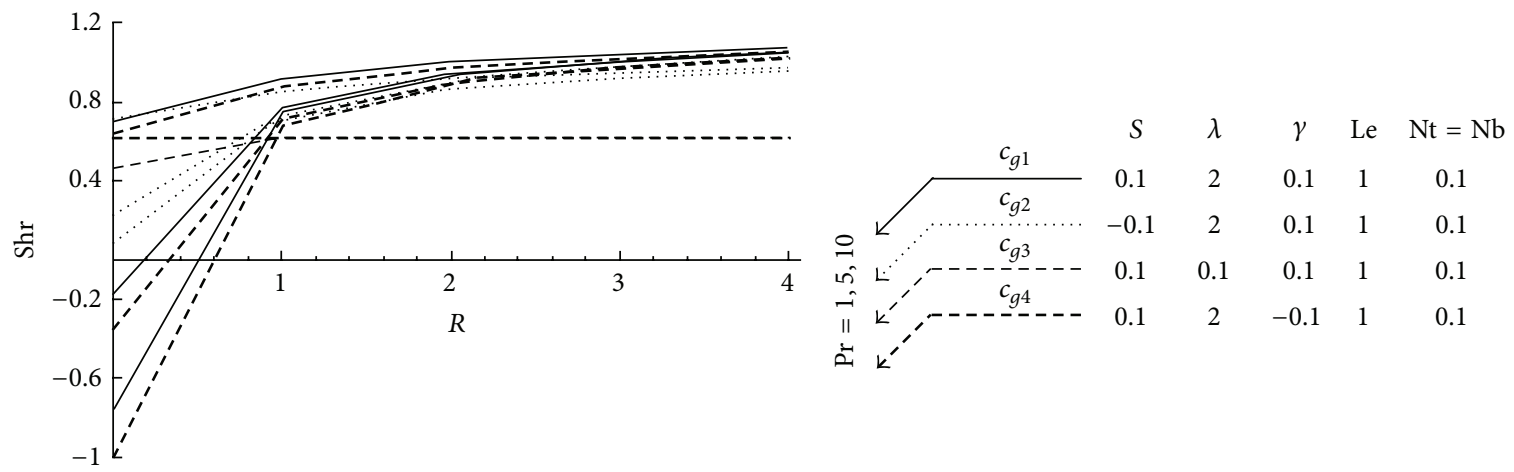

(c)

FIGURE 18: Effects of Nt, Nb, Le, and Pr on relation of reduced Sherwood number [Shr $=-\phi^{\prime}(0)$ ] as a function of the radiation parameter $R$ for $M=K=1, \delta_{1}=1, \delta_{2}=-1$, and $\gamma=0.1$.

\section{Disclosure}

This paper is part of the special issue titled "Macroscopic/Mesoscopic Computational Materials Science Modeling and Engineering (CMSE)".

\section{Conflict of Interests}

The author declares that there is no conflict of interests regarding the publication of this paper.

\section{Acknowledgment}

This work was funded by the Deanship of Scientific Research (DSR), King Abdulaziz University, Jeddah, under Grant no. (965-96-D1435). The author, therefore, acknowledges with thanks DSR technical and financial support.

\section{References}

[1] M. V. Karwe and Y. Jaluria, "Numerical simulation of thermal transport associated with a continuously moving flat sheet in 
materials processing," Journal of Heat Transfer, vol. 113, no. 3, pp. 612-619, 1991.

[2] V. Kumaran, A. V. Kumar, and I. Pop, "Transition of MHD boundary layer flow past a stretching sheet," Communications in Nonlinear Science and Numerical Simulation, vol. 15, no. 2, pp. 300-311, 2010.

[3] S. U. S. Choi, "Enhancing thermal conductivity of fluids with nanoparticles," in Proceedings of the 1995 ASME International Mechanical Engineering Congress and Exposition, vol. 66, pp. 99-105, ASME, FED 231/MD, San Francisco, Calif, USA, 1995.

[4] S. U. S. Choi, Z. G. Zhang, W. Yu, F. E. Lockwood, and E. A. Grulke, "Anomalous thermal conductivity enhancement in nanotube suspensions," Applied Physics Letters, vol. 79, no. 14, pp. 2252-2254, 2001.

[5] H. Masuda, A. Ebata, K. Teramae, and N. Hishinuma, "Alteration of thermal conductivity and viscosity of liquid by dispersing ultra-fine particles. Dispersion of $\mathrm{Al}_{2} \mathrm{O}_{3}, \mathrm{SiO}_{2}$ and $\mathrm{TiO}_{2}$ ultra-fine particles," Netsu Bussei, vol. 7, no. 4, pp. 227-233, 1993.

[6] S. Lee, S. U.-S. Choi, S. Li, and J. A. Eastman, "Measuring thermal conductivity of fluids containing oxide nanoparticles," Journal of Heat Transfer, vol. 121, no. 2, pp. 280-288, 1999.

[7] Y. Xuan and Q. Li, "Heat transfer enhancement of nanofluids," International Journal of Heat and Fluid Flow, vol. 21, no. 1, pp. 58-64, 2000.

[8] Y. Xuan and W. Roetzel, "Conceptions for heat transfer correlation of nanofluids," International Journal of Heat and Mass Transfer, vol. 43, no. 19, pp. 3701-3707, 2000.

[9] M. T. Matthews and J. M. Hill, "A note on the boundary layer equations with linear slip boundary condition," Applied Mathematics Letters, vol. 21, no. 8, pp. 810-813, 2008.

[10] R. A. Van Gorder, E. Sweet, and K. Vajravelu, "Nano boundary layers over stretching surfaces," Communications in Nonlinear Science and Numerical Simulation, vol. 15, no. 6, pp. 1494-1500, 2010.

[11] F. T. Akyildiz, H. Bellout, K. Vajravelu, and R. A. van Gorder, "Existence results for third order nonlinear boundary value problems arising in nano boundary layer fluid flows over stretching surfaces," Nonlinear Analysis: Real World Applications, vol. 12, no. 6, pp. 2919-2930, 2011.

[12] S. Kakaç and A. Pramuanjaroenkij, "Review of convective heat transfer enhancement with nanofluids," International Journal of Heat and Mass Transfer, vol. 52, no. 13-14, pp. 3187-3196, 2009.

[13] P. Rana and R. Bhargava, "Flow and heat transfer of a nanofluid over a nonlinearly stretching sheet: a numerical study," Communications in Nonlinear Science and Numerical Simulation, vol. 17, no. 1, pp. 212-226, 2012.

[14] E. H. Aly and A. Ebaid, "Exact analytical solution for suction and injection flow with thermal enhancement of five nanofluids over an isothermal stretching sheet with effect of the slip model: a comparative study," Abstract and Applied Analysis, vol. 2013, Article ID 721578, 14 pages, 2013.

[15] J. Buongiorno, "Convective transport in nanofluids," Journal of Heat Transfer, vol. 128, no. 3, pp. 240-250, 2006.

[16] T. R. Mahapatra and A. S. Gupta, "Heat transfer in stagnationpoint flow towards a stretching sheet," Heat and Mass Transfer, vol. 38, no. 6, pp. 517-521, 2002.

[17] A. Ishak, K. Jafar, R. Nazar, and I. Pop, "MHD stagnation point flow towards a stretching sheet," Physica A: Statistical Mechanics and its Applications, vol. 388, no. 17, pp. 3377-3383, 2009.

[18] G. C. Layek, S. Mukhopadhyay, and S. K. A. Samad, "Heat and mass transfer analysis for boundary layer stagnationpoint flow towards a heated porous stretching sheet with heat absorption/generation and suction/blowing," International Communications in Heat and Mass Transfer, vol. 34, no. 3, pp. 347-356, 2007.

[19] N. Bachok, A. Ishak, R. Nazar, and I. Pop, "Flow and heat transfer at a general three-dimensional stagnation point in a nanofluid," Physica B: Condensed Matter, vol. 405, no. 24, pp. 4914-4918, 2010.

[20] M. Mustafa, T. Hayat, I. Pop, S. Asghar, and S. Obaidat, "Stagnation-point flow of a nanofluid towards a stretching sheet," International Journal of Heat and Mass Transfer, vol. 54, no. 25-26, pp. 5588-5594, 2011.

[21] M. A. A. Hamad and M. Ferdows, "Similarity solution of boundary layer stagnation-point flow towards a heated porous stretching sheet saturated with a nanofluid with heat absorption/generation and suction/blowing: a Lie group analysis," Communications in Nonlinear Science and Numerical Simulation, vol. 17, no. 1, pp. 132-140, 2012.

[22] L. Zheng, C. Zhang, X. Zhang, and J. Zhang, "Flow and radiation heat transfer of a nanofluid over a stretching sheet with velocity slip and temperature jump in porous medium," Journal of the Franklin Institute. Engineering and Applied Mathematics, vol. 350, no. 5, pp. 990-1007, 2013.

[23] W. Ibrahim, B. Shankar, and M. M. Nandeppanavar, "MHD stagnation point flow and heat transfer due to nanofluid towards a stretching sheet," International Journal of Heat and Mass Transfer, vol. 56, no. 1-2, pp. 1-9, 2013.

[24] B. Jalilpour, S. Jafarmadar, D. D. Ganji, A. B. Shotorban, and H. Taghavifar, "Heat generation/absorption on MHD stagnation flow of nanofluid towards a porous stretching sheet with prescribed surface heat flux," Journal of Molecular Liquids, vol. 195, pp. 194-204, 2014.

[25] P. A. Thompson and S. M. Troian, "A general boundary condition for liquid flow at solid surfaces," Nature, vol. 389, no. 6649, pp. 360-362, 1997.

[26] M. Majumder, N. Chopra, R. Andrews, and B. J. Hinds, "Nanoscale hydrodynamics: enhanced flow in carbon nanotubes," Nature, vol. 438, no. 7064, p. 44, 2005.

[27] A. Noghrehabadi, R. Pourrajab, and M. Ghalambaz, "Effect of partial slip boundary condition on the flow and heat transfer of nanofluids past stretching sheet prescribed constant wall temperature," International Journal of Thermal Sciences, vol. 54, pp. 253-261, 2012.

[28] W. A. Khan and I. Pop, "Boundary-layer flow of a nanofluid past a stretching sheet," International Journal of Heat and Mass Transfer, vol. 53, no. 11-12, pp. 2477-2483, 2010.

[29] M. M. Nandeppanavar, K. Vajravelu, M. S. Abel, and M. N. Siddalingappa, "Second order slip flow and heat transfer over a stretching sheet with non-linear Navier boundary condition," International Journal of Thermal Sciences, vol. 58, pp. 143-150, 2012.

[30] T. Fang, S. Yao, J. Zhang, and A. Aziz, "Viscous flow over a shrinking sheet with a second order slip flow model," Communications in Nonlinear Science and Numerical Simulation, vol. 15, no. 7, pp. 1831-1842, 2010.

[31] L. A. Wu, "A slip model for rarefied gas flows at arbitrary Knudsen number," Applied Physics Letters, vol. 93, no. 25, Article ID 253103, 2008.

[32] M. Turkyilmazoglu, "Heat and mass transfer of MHD second order slip flow," Computers \& Fluids, vol. 71, pp. 426-434, 2013.

[33] A. V. Roşca and I. Pop, "Flow and heat transfer over a vertical permeable stretching/shrinking sheet with a second order slip," 
International Journal of Heat and Mass Transfer, vol. 60, no. 1, pp. 355-364, 2013.

[34] E. H. Aly and K. Vajravelu, "Exact and numerical solutions of MHD nano boundary-layer flows over stretching surfaces in a porous medium," Applied Mathematics and Computation, vol. 232, pp. 191-204, 2014.

[35] E. H. Aly and M. A. Hassan, "Suction and injection analysis of mhd nano boundary-layer over a stretching surface through a porous medium with partial slip boundary condition," Journal of Computational and Theoretical Nanoscience, vol. 11, no. 3, pp. 827-839, 2014.

[36] D. A. Nield and A. V. Kuznetsov, "The Cheng-Minkowycz problem for natural convective boundary-layer flow in a porous medium saturated by a nanofluid," International Journal of Heat and Mass Transfer, vol. 52, no. 25-26, pp. 5792-5795, 2009.

[37] M. Q. Brewster, Thermal Radiative Transfer Properties, John Wiley and Sons, New York, NY, USA, 1992.

[38] R. C. Bataller, "Radiation effects for the Blasius and Sakiadis flows with a convective surface boundary condition," Applied Mathematics and Computation, vol. 206, no. 2, pp. 832-840, 2008.

[39] K. Vajravelu, K. V. Prasad, J. Lee, C. Lee, I. Pop, and R. A. van Gorder, "Convective heat transfer in the flow of viscous Ag-water and $\mathrm{Cu}$-water nanofluids over a stretching surface," International Journal of Thermal Sciences, vol. 50, no. 5, pp. 843851, 2011.

[40] E. H. Aly, "Effect of the velocity slip boundary condition on the flow and heat transfer of nanofluids over a stretching sheet," Journal of Computational and Theoretical Nanoscience, vol. 12, no. 10, pp. 1-9, 2015.

[41] E. H. Aly and H. M. Sayed, "Thermal radiation effects on magnetohydrodynamic boundary-layer flow due to a moving extensible surface in nanofluids," Journal of Computational and Theoretical Nanoscience, vol. 11, no. 8, pp. 1756-1765, 2014.

[42] T. Hayat, T. Javed, and Z. Abbas, "MHD flow of a micropolar fluid near a stagnation-point towards a non-linear stretching surface," Nonlinear Analysis: Real World Applications, vol. 10, no. 3, pp. 1514-1526, 2009.

[43] C. Y. Wang, "Free convection on a vertical stretching surface," Journal of Applied Mathematics and Mechanics (ZAMM), vol. 69, no. 11, pp. 418-420, 1989. 


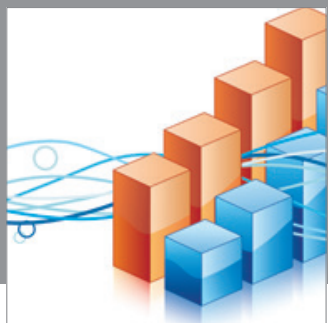

Advances in

Operations Research

mansans

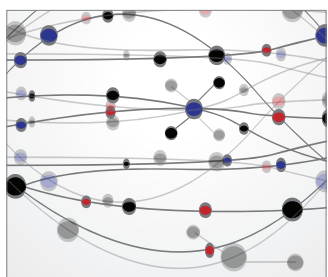

The Scientific World Journal
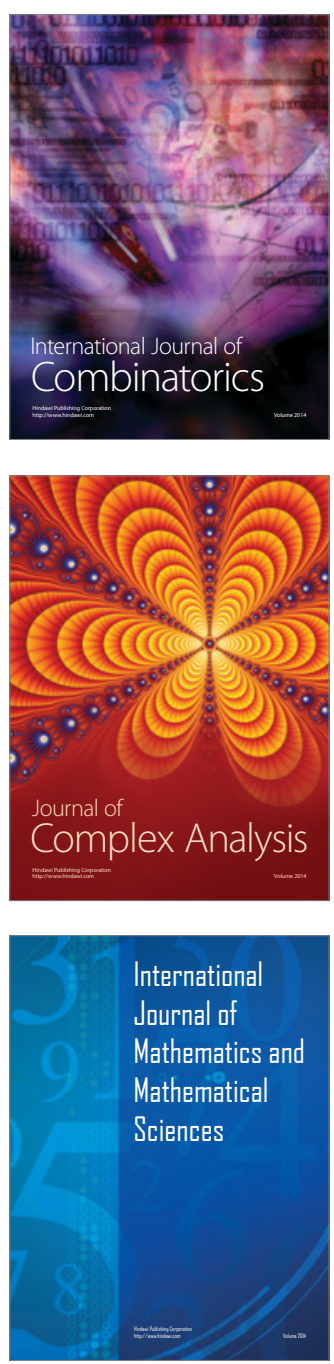
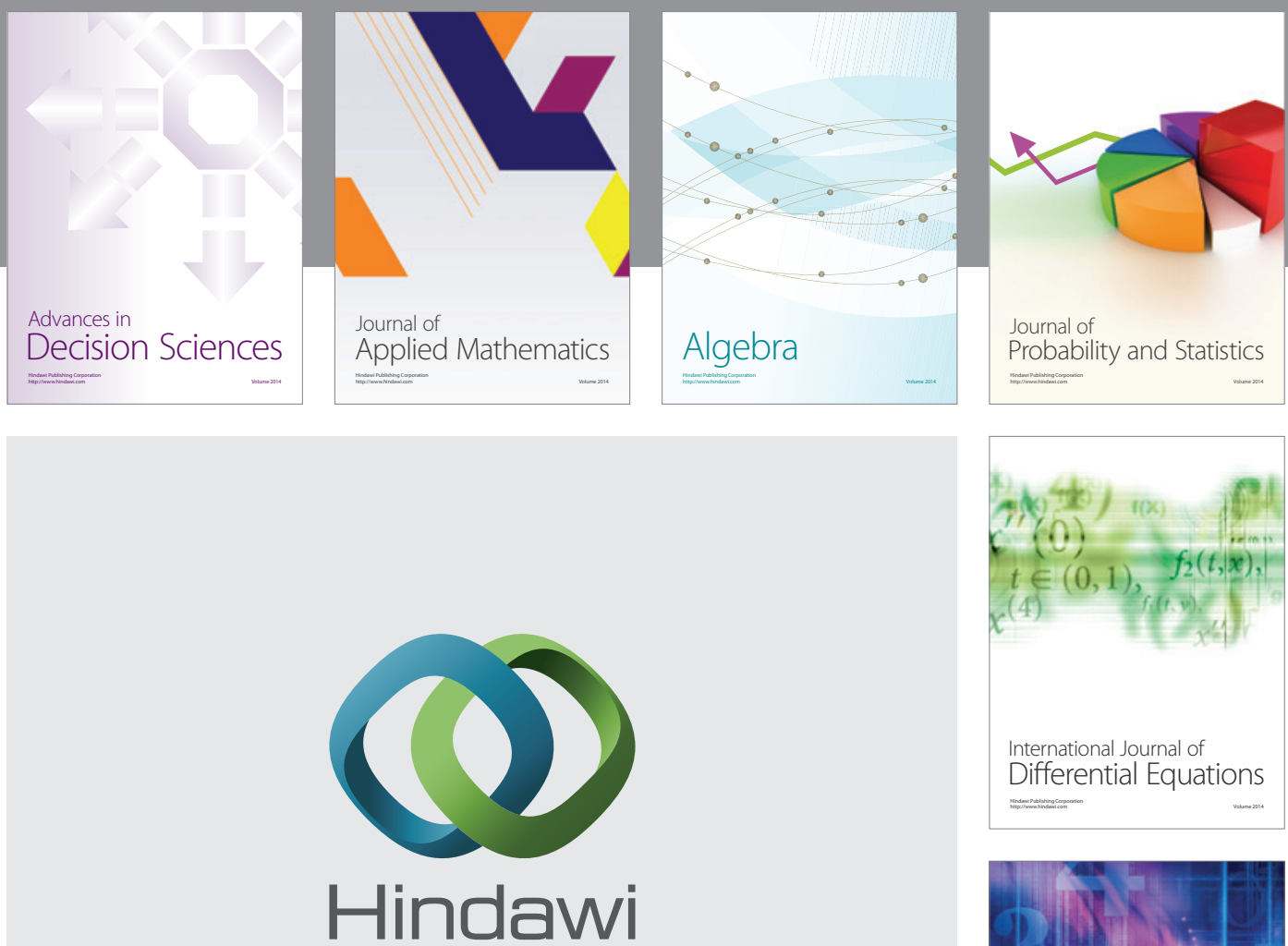

Submit your manuscripts at http://www.hindawi.com
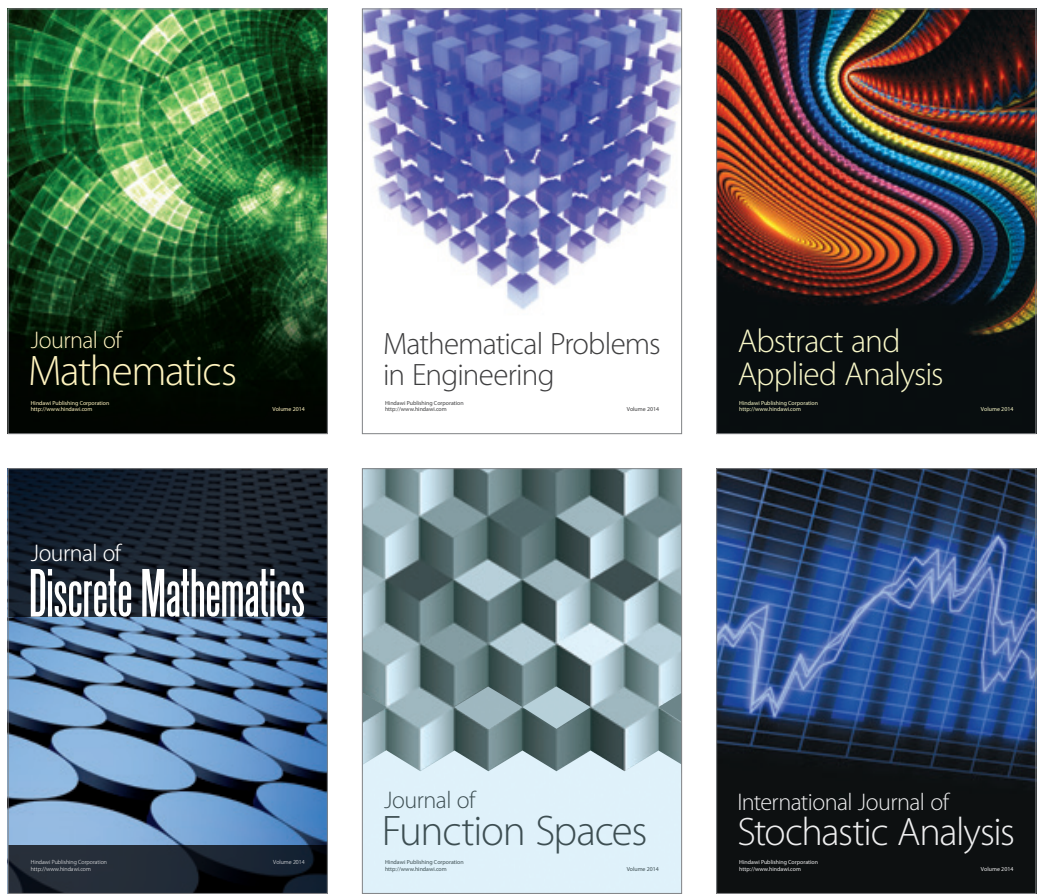

Journal of

Function Spaces

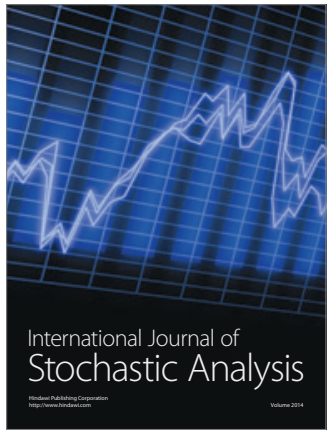

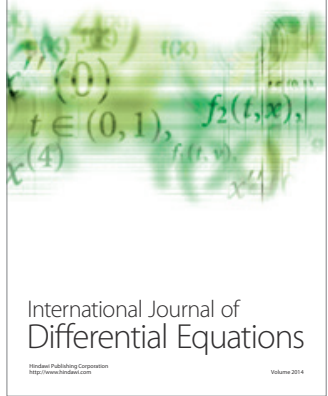
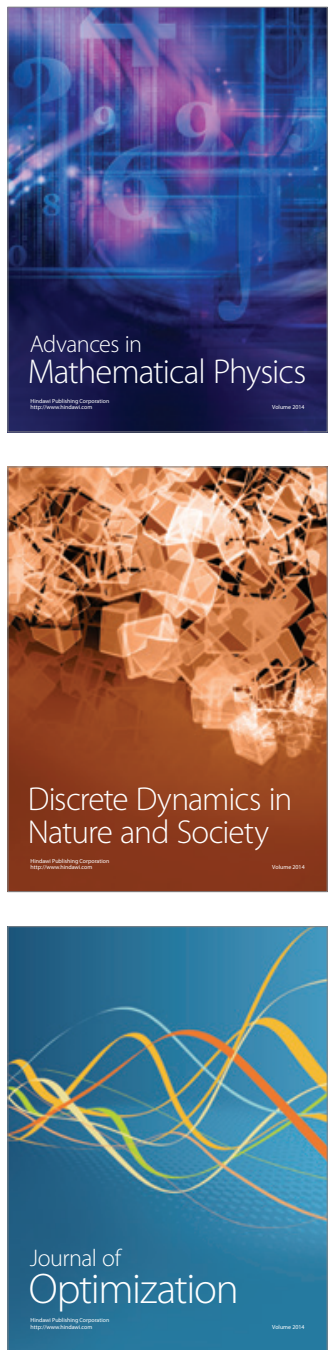